Pacific

Journal of

Mathematics

FLATNESS AND FUSION COEFFICIENTS

ViJay Kodiyalam and V.S. SUnder 


\title{
FLATNESS AND FUSION COEFFICIENTS
}

\author{
Vijay Kodiyalam And V.S. Sunder
}

\begin{abstract}
This paper addresses the problem of how to directly read off the 'fusion coefficients' (for the tensor-products of bimodules arising in the 'tower of the basic construction') for a subfactor, from the data of the associated paragroup. The solution to this problem is closely related to a reformulation, which is obtained here, of the flatness condition to be satisfied by the connection on a paragroup; our reformulation is best described by the phrase ' $1 *$ flatness', in contrast with Ocneanu's initial formulation as ' $4 *$ flatness' and Kawahigashi's subsequent reformulation as ' $2 *$ flatness'.
\end{abstract}

\section{Introduction.}

Ocneanu obtained — see [AO1], [AO2] — a combinatorial structure, which he called a paragroup, as a complete isomorphism-invariant for the so-called 'finite-depth subfactors of the hyperfinite $\mathrm{II}_{1}$ factor', which has been quite useful in various related considerations. As stated in the abstract, in this paper, we obtain formulae for the fusion coefficients for tensor-products of the four kinds of bimodules associated to a finite-depth hyperfinite subfactor. Most existing computations of such fusion rules use only the principal and dual principal graphs of the subfactor, by some ad hoc methods; but as Bisch found - see [DB] - there exist five different fusion algebras that are consistent with the graphs of the $3+\sqrt{3}$ subfactor. This is the reason we wished to find a formula that derives the fusion coefficients using only the standard invariant (which must necessarily involve the underlying flat connection).

It should probably be mentioned that the literature contains various other treatments of the fusion rule algebra of a subfactor - for instance, see $[\mathbf{I z}]$, [FRS], [Lo], [Lo1] (the endomorphism/sector approach), [DB], [DB1] (the bimodule-shift-projection approach), [GW] (the shift-projection approach in the special case of Hecke algebra subfactors), [SV] (the bimodule approach), etc. It should definitely be mentioned that the authors only became aware of the work [Gne], which has nontrivial overlap with this paper, after these results had been obtained. ${ }^{1}$

\footnotetext{
${ }^{1}$ We would like to thank Kawahigashi for pointing out this reference to us. (In fact, he himself had initially acknowledged being unaware of any work along these lines until Sato had brought this work to his notice.)
} 
The paper is organised as follows.

In $\S 2$, we recall the basic notions - connection on a suitable pair of bipartite graphs, unitarity and 'flip-invariance' of the connection, flatness, etc. - needed to describe paragroups. (It should be remarked, however, that our description and notation for a paragroup vary somewhat from the one most commonly found in the literature (as in $[\mathbf{E K}]$ or $[\mathbf{Y K}])$.)

In $\S 3$, we first deduce our ' $1 *$ flatness' condition from the $(4 *)$ flatness axiom on the paragroup, and then derive some consequences of this condition, including the fact that the ' $4 *$ flatness' axiom may be deduced from the $1 *$ version.

In $\S 4$, we show that a certain function that comes up naturally in the context of ' $1 *$ flatness' is actually nothing but the fusion coefficients of the associated subfactor. We recall various facts concerning path models which are used in the proof of this assertion.

In $\S 5$, we show how one can directly (without having to go through the associated subfactor and relying on the bijectivity of the pairing between paragroups and 'finite-depth hyperfinite subfactors') show that the paragoup gives rise to a pair of fusion algebras, as well as the known fact (see [EK]) that of the four graphs appearing in the definition of the paragroup, there are natural isomorphisms between two pairs.

\section{Paragroups.}

We recall here what goes into Ocneanu's paragroup. The basic ingredient is a collection $\left\{V_{0}, V_{1}, H_{0}, H_{1}\right\}$ of graphs (where we allow multiple bonds between the same pair of vertices, but do NOT allow loops); we shall refer to the $V_{i}$ 's (resp., the $H_{j}$ 's) as the vertical (resp., horizontal) graphs, and to edges in vertical (resp., horizontal) graphs as vertical (resp., horizontal) edges.

The graphs are required to satisfy the following requirements:

Vertex requirements:

(i) (Vertex identifications):

Each of the four graphs is a finite, connected bipartite graph; further, it is assumed that there are four sets $\left\{B_{i j}: 0 \leq i, j \leq 1\right\}$ such that the decomposition of the vertex sets of $V_{0}$ and $V_{1}$ (resp., $H_{0}$ and $H_{1}$ ) are given by $B_{00} \coprod B_{10}$ and $B_{01} \amalg B_{11}$ (resp., $B_{00} \amalg B_{01}$ and $B_{10} \coprod B_{11}$ ) respectively. (Here and elsewhere, the symbol $\amalg$ denotes 'disjoint union'.)

(ii) (* vertices and $\alpha$ vertices):

There exist vertices $*_{0} \in B_{00}, *_{1} \in B_{11}$ and $\alpha_{01} \in B_{01}, \alpha_{10} \in B_{10}$ such that: 
(a) $*_{0}$ has degree 1 in $V_{0}$ as well as $H_{0}$; and $\alpha_{10}$ (resp., $\alpha_{01}$ ) is the only vertex which is adjacent to $*_{0}$ in $V_{0}$ (resp., $H_{0}$ ); and

(b) $*_{1}$ has degree 1 in $V_{1}$ as well as $H_{1}$; and $\alpha_{01}$ (resp., $\alpha_{10}$ ) is the only vertex which is adjacent to $*_{1}$ in $V_{1}$ (resp., $H_{1}$ ).

\section{Harmonicity:}

There exists a function $\mu: \coprod_{i, j=0,1} B_{i j} \rightarrow(0, \infty)$, and a scalar $\beta>0$ which satisfy:

(i) $\mu\left(*_{i}\right)=1$ for $i=0,1$; and

(ii) let $G$ denote any of the four graphs above. If $A_{G}$ denotes the adjacency matrix of $G$ (defined by letting $A_{G}(u, v)$ denote the number of edges joining the vertices $u$ and $v$ ), then

$$
\sum_{\lambda} A_{G}(\pi, \lambda) \mu(\lambda)=\beta \mu(\pi)
$$

for all vertices $\pi$ of the graph.

(Thus $\beta$ is the Perron-Frobenius eigenvalue - see [FRG], for instance of (the adjacency matrix of) each of the four graphs, and the corresponding Perron-Frobenius eigenvectors (uniquely determined by the normalisation (i) above) are required to be consistent with the vertex identifications, and they 'patch up' to give the function $\mu$.)

We shall henceforth use the 'subscript convention' whereby a symbol such as $\gamma_{p q}$ will always denote a vertex in $B_{p q}$; further, subscripts such as $i, j, p, q$ will always be assumed to range over $\{0,1\}$.

The connection:

By a cell is meant a configuration of the form

$$
\begin{array}{ccc}
\gamma_{i, j} & \tau_{i} & \gamma_{i, 1-j} \\
\sigma_{j} & & \sigma_{1-j} \\
\gamma_{1-i, j} & \tau_{1-i} & \gamma_{1-i, 1-j}
\end{array}
$$

where it is assumed that (a) $\tau_{p}$ is an edge joining $\gamma_{p, j}$ and $\gamma_{p, 1-j}$ in $H_{p}$, for $p=i, 1-i$; and (b) $\sigma_{q}$ is an edge joining $\gamma_{i, q}$ and $\gamma_{1-i, q}$ in $V_{q}$, for $q=$ $j, 1-j$.

The connection is a function, which assigns a complex number to each cell as above; the number associated by the connection to the cell illustrated in (2.1) will be denoted by either of the following symbols:

$$
W_{\tau_{i} \sigma_{1-j}}^{\sigma_{j} \tau_{1-i}}=\begin{array}{cccc}
\gamma_{i, j} & \stackrel{\tau_{i}}{\rightarrow} & \gamma_{i, 1-j} \\
\sigma_{j} \downarrow & & \downarrow \sigma_{1-j} \\
\gamma_{1-i, j} & \stackrel{\tau_{1-i}}{\rightarrow} & \gamma_{1-i, 1-j}
\end{array} .
$$

(We shall refer to $\sigma_{j} \tau_{1-i}$ and $\tau_{i} \sigma_{1-j}$ as the south-west and north-east paths, respectively, of the cell depicted in (2.1). The $W$-notation for the connection is meant to indicate that this value of the connection is to be 
regarded as the entry of a matrix with rows indexed by appropriate southwest paths and columns indexed by appropriate north-east paths.)

The connection is required to satisfy the following conditions:

Unitarity: For any pair $\gamma_{i j}, \gamma_{1-i, 1-j}$ of vertices, the matrix

$$
W\left(\gamma_{i j}, \gamma_{1-i, 1-j}\right)=\stackrel{\gamma_{i, j}-}{\mid} \quad \gamma_{1-i, 1-j},
$$

with rows (resp., columns) indexed by south-west (resp., north-east) paths from $\gamma_{i j}$ to $\gamma_{1-i, 1-j}$, is a unitary matrix.

Invariance under flips: With the notation of (2.2), we have:

$$
\begin{aligned}
& \begin{array}{ccc}
\gamma_{i, j} & \stackrel{\tau_{i}}{\rightarrow} & \gamma_{i, 1-j} \\
\sigma_{j} \downarrow & & \downarrow \sigma_{1-j} \\
\gamma_{1-i, j} & \stackrel{\tau_{1-i}}{\rightarrow} & \gamma_{1-i, 1-j}
\end{array} \\
& =\sqrt{\frac{\mu\left(\gamma_{1-i, j}\right) \mu\left(\gamma_{i, 1-j}\right)}{\mu\left(\gamma_{i j}\right) \mu\left(\gamma_{1-i, 1-j}\right)}}\left(\begin{array}{ccc}
\gamma_{i, 1-j} & \stackrel{\tau_{i}}{\rightarrow} & \gamma_{i, j} \\
\sigma_{1-j} \downarrow & & \downarrow \sigma_{j} \\
\gamma_{1-i, 1-j} & \stackrel{\tau_{1-i}}{\rightarrow} & \gamma_{1-i, j}
\end{array}\right)^{-} \\
& =\sqrt{\frac{\mu\left(\gamma_{1-i, j}\right) \mu\left(\gamma_{i, 1-j}\right)}{\mu\left(\gamma_{i j}\right) \mu\left(\gamma_{1-i, 1-j}\right)}}\left(\begin{array}{ccc}
\gamma_{1-i, j} & \stackrel{\tau_{1-i}}{\longrightarrow} & \gamma_{1-i, 1-j} \\
\sigma_{j} \downarrow & & \downarrow \sigma_{1-j} \\
\gamma_{i, j} & \stackrel{\tau_{i}}{\rightarrow} & \gamma_{i, 1-j}
\end{array}\right)^{-} \\
& =\begin{array}{ccc}
\gamma_{1-i, 1-j} & \stackrel{\tau_{1-i}}{\rightarrow} & \gamma_{1-i, j} \\
\sigma_{1-j} \downarrow & & \downarrow \sigma_{j} \\
\gamma_{i, 1-j} & \stackrel{\tau_{i}}{\rightarrow} & \gamma_{i, j} .
\end{array}
\end{aligned}
$$

(The first two equations state 'invariance' of the connection under 'horizontal flips' and 'vertical flips' respectively, while the third equation (which is a consequence of the previous two) states a 'rotational symmetry' of the connection.)

Before proceeding to the final - and most crucial — 'flatness' requirement to be satisfied by a connection, we need to make some comments about paths and larger rectangles.

Recall that a path of length $k$ in a graph is a sequence

$$
\sigma=\left(v_{0}, \sigma_{1}, v_{1}, \cdots, v_{r-1}, \sigma_{r}, v_{r}, \cdots, v_{k-1}, \sigma_{k}, v_{k}\right),
$$

where $\sigma_{r}$ is an edge joining $v_{r-1}$ and $v_{r}$, for $1 \leq r \leq k$. We shall say that $\sigma$ starts at $v_{0}$ and ends at $v_{k}$. We shall also use the notation $\sigma^{o}$ to denote the opposite or reversed path (which starts at $v_{k}$ and finishes at $v_{0}$ ).

The symbol $\tau$ (resp., $\sigma$ ) will be reserved for a horizontal (resp., vertical) path. 
We shall use the following notation: The symbol $\mathcal{P}_{l}^{H}(\pi, \lambda)\left(\operatorname{resp} ., \mathcal{P}_{k}^{V}(\pi, \lambda)\right)$ will denote the collection of horizontal (resp., vertical) paths of length $l$ (resp., $k$ ) between the vertices $\pi$ and $\lambda$. Also, we shall write $|\sigma|$ for the length of $\sigma$, as well as $\mathcal{P}_{k}^{V}(\pi,-)$ for the set of vertical paths of length $k$ starting at $\pi$, and $\mathcal{P}^{V}(\pi, \lambda)$ for the set of vertical paths of arbitrary length between $\pi$ and $\lambda$, as well as corresponding 'horizontal' versions.

We shall need to work with the following generalised cells, which we follow Ocneanu in calling macro-cells:

$$
\begin{array}{ccc}
\gamma_{p r} & \tau_{p} & \gamma_{p s} \\
\sigma_{r} & & \sigma_{s} \\
\gamma_{q r} & \tau_{q} & \gamma_{q s}
\end{array},
$$

where (a) $\tau_{i}$ is a horizontal path from $\gamma_{i r}$ to $\gamma_{i s}$ and $\sigma_{j}$ is a vertical path from $\gamma_{p j}$ to $\gamma_{q j}$, for $j \in\{r, s\}, i \in\{p, q\}$, and (b) $\left|\tau_{p}\right|=\left|\tau_{q}\right|$, and $\left|\sigma_{r}\right|=\left|\sigma_{s}\right|$.

When dealing with a macro-cell as above, we shall always regard the horizontal paths as 'going from left to right' and the vertical paths as 'going from top to bottom'.

Given such a macro-cell, we associate a connection-value, denoted by

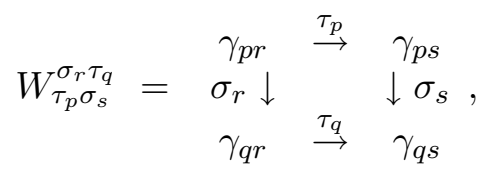

as follows.

Rather than giving the general definition, we shall describe it in a special case. Consider the case when the horizontal paths have length 3 and the horizontal paths have length 2 ; then, we define

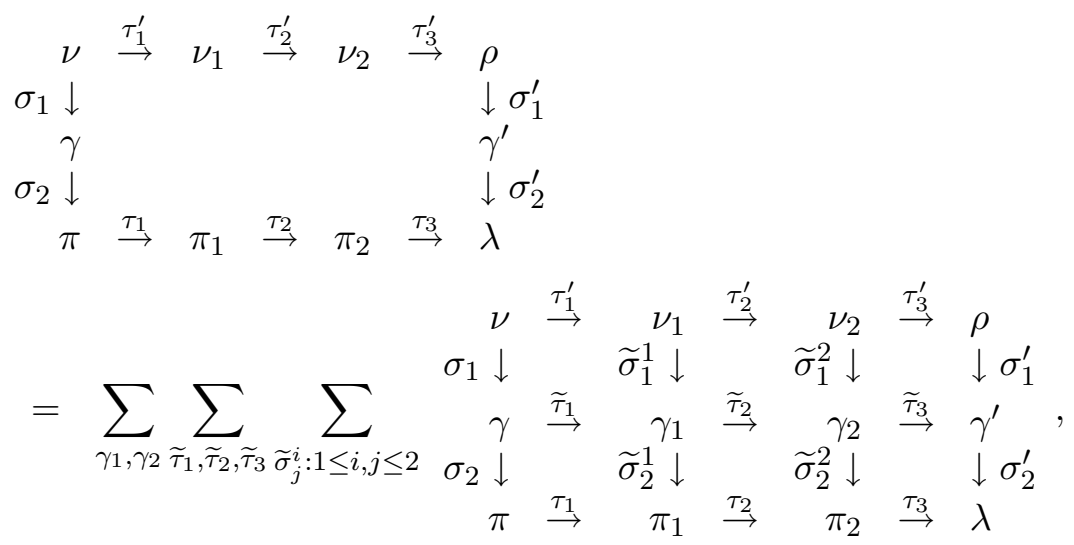

where:

(i) $\gamma_{1}$ is any vertex that is simultaneously a 'horizontal neighbour' of $\gamma$ as well as a 'vertical neighbour' of both $\nu_{1}$ and $\pi_{1}$;

(ii) $\gamma_{2}$ is any vertex that is simultaneously a 'horizontal neighbour' of both $\gamma_{1}$ and $\gamma^{\prime}$, as well as a 'vertical neighbour' of both $\nu_{2}$ and $\pi_{2}$; 


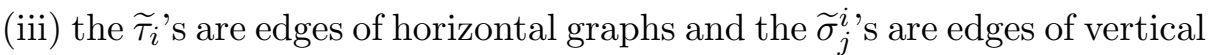
graphs, with end-points as indicated;

(iv) the value of the totally labelled ' $2 \times 3$ ' rectangle in the second line of the displayed equation above, is interpreted as the product of the values assigned by the connection to each of the six ' $1 \times 1$ ' subrectangles.

It is true, and not very hard to verify, that for the configuation described by the general macro-cell shown in (2.3), with $\left|\tau_{p}\right|=\left|\tau_{q}\right|=l$ (say), and $\left|\sigma_{r}\right|=$ $\left|\sigma_{s}\right|=k$ (say), if we consider the matrix $W_{k l}\left(\gamma_{p r}, \gamma_{q s}\right)=\left(\left(W_{\tau_{p} \sigma_{s}}^{\sigma_{r} \tau_{q}}\right)\right)$, with rows indexed by 'south-west paths of type $(k, l)$ ' (through arbitrary $\gamma_{q r}$ ) and columns indexed by 'north-east paths of type $(l, k)$ ' (through arbitrary $\gamma_{p s}$ ), from $\gamma_{p r}$ to $\gamma_{q s}$, then this matrix is unitary; and further, this extension of the connection to 'macro-cells' also satisfies an appropriately amended version of the 'invariance under flips' condition, as follows:

$$
\begin{aligned}
& \begin{array}{lll}
\gamma_{i, j} & \stackrel{\tau_{i}}{\rightarrow} & \gamma_{i, r} \\
\sigma_{j} \downarrow & & \downarrow \sigma_{r} \\
\gamma_{p, j} & \stackrel{\tau_{p}}{\rightarrow} & \gamma_{p, r}
\end{array} \\
& =\sqrt{\frac{\mu\left(\gamma_{p, j}\right) \mu\left(\gamma_{i, r}\right)}{\mu\left(\gamma_{i, j}\right) \mu\left(\gamma_{p, r}\right)}}\left(\begin{array}{ccc}
\gamma_{i, r} & \stackrel{\tau_{i}^{o}}{\rightarrow} & \gamma_{i, j} \\
\sigma_{r} \downarrow & & \downarrow \sigma_{j} \\
\gamma_{p, r} & \stackrel{\tau_{p}^{o}}{\rightarrow} & \gamma_{p, j}
\end{array}\right)^{-} \\
& =\sqrt{\frac{\mu\left(\gamma_{p, j}\right) \mu\left(\gamma_{i, r}\right)}{\mu\left(\gamma_{i, j}\right) \mu\left(\gamma_{p, r}\right)}}\left(\begin{array}{ccc}
\gamma_{p, j} & \stackrel{\tau_{p}}{\rightarrow} & \gamma_{p, r} \\
\sigma_{j}^{o} \downarrow & & \downarrow \sigma_{r}^{o} \\
\gamma_{i, j} & \stackrel{\tau_{i}}{\rightarrow} & \gamma_{i, r}
\end{array}\right)^{-} \\
& \begin{array}{cccc}
\gamma_{p, r} & \stackrel{\tau_{p}^{o}}{\rightarrow} & \gamma_{p, j} \\
\sigma_{r}^{o} \downarrow & & \downarrow \sigma_{j}^{o} \\
\gamma_{i, r} & \stackrel{\tau_{i}^{o}}{\rightarrow} & \gamma_{i, j}
\end{array} .
\end{aligned}
$$

We are now ready for the 'flatness' condition.

\section{Flatness axiom:}

Let $*$ denote either $*_{0}$ or $*_{1}$ (in all occurrences of $*$ below). Then, for any (necessarily even) positive integers $k, l$, and vertical paths $\sigma, \sigma^{\prime} \in \mathcal{P}_{k}^{V}(*, *)$ and horizontal paths $\tau, \tau^{\prime} \in \mathcal{P}_{l}^{H}(*, *)$, we have:

$$
\begin{array}{rll}
\sigma & \stackrel{\tau}{\rightarrow} & * \\
* & \stackrel{\tau^{\prime}}{\rightarrow} & \downarrow
\end{array}
$$

Definition 2.1. A coupled system is the data $\left(V_{0}, V_{1}, H_{0}, H_{1} ; W\right)$ consisting of a collection of four finite, connected, bipartite graphs and a connection 
$W$ defined on them, which satisfy the six requirements that we have termed vertex identification, $*$ vertices and $\alpha$ vertices, harmonicity, unitarity, invariance under flips, and the flatness axiom.

It is possible to define an equivalence relation on the class of coupled systems; this involves changing the graphs up to graph isomorphisms (which 'patch up' well) and changing the connection up to a 'coboundary'. We shall not make this any more precise, since we will not need the exact definition. (The interested reader may consult $[\mathbf{A O 1}],[\mathbf{E K}]$ or $[\mathbf{J S}]$.)

A paragroup is an equivalence class of coupled systems.

Remark 2.2. It must be noted that we only discuss finite paragroups here. Also, all other structure of the paragroup (such as the 'global contragredient axiom') will be shown to be consequences of our definition - at least in this finite case.

In fact, more general 'unitarity statements' (than in the paragraph preceding the flatness axiom) hold for any connection which satisfies the unitarity condition; we need to set up a little notation in order to state this generalisation.

Fix a positive integer $n$; we shall use the phrase admissible tuple of size $n$ to mean an ordered pair $(\mathbf{k}, \mathbf{l})$, where

$$
\mathbf{k}=\left(k_{1}, k_{2}, \cdots, k_{n}\right), \mathbf{l}=\left(l_{1}, l_{2}, \cdots, l_{n}\right)
$$

and the $k_{i}$ and $l_{j}$ are all nonnegative integers, of which only $k_{1}$ and $l_{n}$ may be zero. An admissible tuple $(\mathbf{k}, \mathbf{l})$ of size $n$ determines a planar walk on the (planar) lattice by the following prescription: Start at the origin; take $k_{1}$ steps to the south; then take $l_{1}$ steps east; then take $k_{2}$ steps south; then take $l_{2}$ steps east; and so on, until a total of $\sum_{i}\left(k_{i}+l_{i}\right)$ steps have been taken. (The possibility, that such a walk may not have an initial southward step or a final eastward step, is the reason for the peculiar 'nonzero' assumptions in the definition of an admissible tuple.)

Given such an admissible tuple, we shall use the expression 'path of type $(\mathbf{k}, \mathbf{l})$ from a vertex $\nu$ to a vertex $\lambda^{\prime}$ to mean a sequence

$$
\left(\sigma_{1}, \tau_{1}, \cdots, \sigma_{i}, \tau_{i}, \cdots, \sigma_{n}, \tau_{n}\right),
$$

where (i) each $\sigma_{i}$ (resp., $\tau_{i}$ ) is a vertical (resp., horizontal) path of length $k_{i}$ (resp., $l_{i}$ ), (ii) $\sigma_{1}$ starts at $\nu$ and $\tau_{n}$ finishes at $\lambda$, and (iii) for each $i, \sigma_{i}$ finishes where $\tau_{i}$ starts, and $\tau_{i}$ finishes where $\sigma_{i+1}$ starts.

Given two admissible tuples $(\mathbf{k}, \mathbf{l}),\left(\mathbf{k}^{\prime}, \mathbf{l}^{\prime}\right)$, of possibly different sizes, we shall say that $(\mathbf{k}, \mathbf{l}) \geq\left(\mathbf{k}^{\prime}, \mathbf{l}^{\prime}\right)$ if the following conditions are satisfied:

(i) $\sum_{i} k_{i}=\sum_{i^{\prime}} k_{i^{\prime}}^{\prime}$ and $\sum_{i} l_{i}=\sum_{i^{\prime}} l_{i^{\prime}}^{\prime}$; and

(ii) for each $1 \leq j \leq \sum_{i}\left(k_{i}+l_{i}\right)$, the number of southward steps taken in the first $j$ steps of the planar walk corresponding to $(\mathbf{k}, \mathbf{l})$ is at least as large as the corresponding number for $\left(\mathbf{k}^{\prime}, \mathbf{l}^{\prime}\right)$. 
(This just says that the walk for $(\mathbf{k}, \mathbf{l})$ is always 'below' the walk for $\left(\mathbf{k}^{\prime}, \mathbf{l}^{\prime}\right)$.)

If $(\mathbf{k}, \mathbf{l}) \geq\left(\mathbf{k}^{\prime}, \mathbf{l}^{\prime}\right)$ are admissible tuples, and if $\nu$ and $\lambda$ are arbitrary vertices, consider the matrix $W_{(\mathbf{k}, \mathbf{l}),\left(\mathbf{k}^{\prime}, \mathbf{l}^{\prime}\right)}(\nu, \lambda)=\left(\left(W_{\eta}^{\xi}\right)\right)$, with rows (resp., columns) indexed by paths $\xi$ (resp., $\eta$ ) of type $(\mathbf{k}, \mathbf{l})$ (resp., $\left(\mathbf{k}^{\prime}, \mathbf{l}^{\prime}\right)$ ) from $\nu$ to $\lambda$, whose typical matrix entry is defined as follows: (i) If it so happens that there exists $1 \leq j \leq \sum_{i}\left(k_{i}+l_{i}\right)$ such that the initial and final points (on the lattice) of the $j$-th step in the planar walk for $(\mathbf{k}, \mathbf{l})$ agree with the corresponding points for the walk for $\left(\mathbf{k}^{\prime}, \mathbf{l}^{\prime}\right)$, then $W_{\eta}^{\xi}=0$ unless the $j$-th edge in the path $\xi$ is the same as the $j$-th edge in the path $\eta$; and (ii) $W_{\eta}^{\xi}$ is defined as the sum of products of values of the connection on $1 \times 1$ subrectangles, exactly as in the case of macro-cells (which correspond to the specialisation $\left.(\mathbf{k}, \mathbf{l})=(k, l),\left(\mathbf{k}^{\prime}, \mathbf{l}^{\prime}\right)=((0, k),(l, 0))\right)$.

We state the desired generalisation of unitarity as a lemma below, for convenience of reference; we omit the proof of this well-known fact. (See, for instance, $[\mathbf{A O 2}]$ or $[\mathbf{E K}]$.)

Lemma 2.3. Suppose $(\mathbf{k}, \mathbf{l}) \geq\left(\mathbf{k}^{\prime}, \mathbf{l}^{\prime}\right) \geq\left(\mathbf{k}^{\prime \prime}, \mathbf{l}^{\prime \prime}\right)$ are admissible tuples; and suppose $\nu \in B_{p q}, \lambda \in B_{r s}$ are such that $\sum_{i} k_{i}-(r-p)$ and $\sum_{i} l_{i}-(s-q)$ are even.

(a) Then $W_{(\mathbf{k}, \mathbf{l}),\left(\mathbf{k}^{\prime}, \mathbf{l}^{\prime}\right)}(\nu, \lambda)$ is a unitary matrix; and

(b) $W_{(\mathbf{k}, \mathbf{l}),\left(\mathbf{k}^{\prime}, \mathbf{l}^{\prime}\right)}(\nu, \lambda) W_{\left(\mathbf{k}^{\prime}, \mathbf{l}^{\prime}\right),\left(\mathbf{k}^{\prime \prime}, \mathbf{l}^{\prime \prime}\right)}(\nu, \lambda)=W_{(\mathbf{k}, \mathbf{l}),\left(\mathbf{k}^{\prime \prime}, \mathbf{l}^{\prime \prime}\right)}(\nu, \lambda)$.

\section{3. $1 *$ flatness.}

We would like to use the phrase ' $4 *$ flatness' to refer to what we have termed the flatness axiom above. This is because it is known - see [EK] — that this requirement can be shown to be equivalent to another one of a pair of equivalent requirements, that should analogously be termed ' $2 *$ flatness'.

We wish to talk about ' $1 *$ flatness' — which, besides being equivalent to the ' $4 *$ flatness' condition, has the advantage of being intimately connected with the 'fusion coefficients' between the four kinds of bimodules associated with the subfactor corresponding to the paragroup.

Definition 3.1. Let $*=*_{i}, i=0,1$. Fix positive integers $k, l$, a vertex $\lambda \in B_{p, r}$, and paths $\sigma \in \mathcal{P}_{k}^{V}(*,-)$ and $\tau \in \mathcal{P}_{l}^{H}(*,-)$, where it is assumed that $p-i-l$ and $r-i-k$ are even. Suppose $\sigma$ and $\tau$ finish at $\pi$ and $\rho$, respectively.

We shall write $A(\sigma, \tau, \lambda)$ for the matrix, with rows and columns indexed by $\tau^{\prime} \in \mathcal{P}_{l}^{H}(\pi, \lambda)$ and $\sigma^{\prime} \in \mathcal{P}_{k}^{V}(\rho, \lambda)$ respectively, defined by

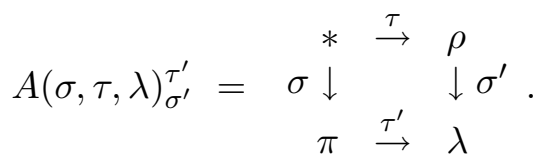


(It must be noted that in order for $A(\sigma, \tau, \lambda)$ to be a nonzero matrix, it is necessary that there exist (necessarily uniquely defined) $i, p, r \in\{0,1\}$ such that $*=*_{i}, \rho \in B_{i r}, \pi \in B_{p i}$ and $\left.\lambda \in B_{p r}.\right)$

Proposition 3.2 ( $1 *$ flatness). Suppose $\sigma_{i}$ are vertical paths starting at $*$ and $\tau_{i}$ are horizontal paths starting at $*$, for $i=1,2,3$, which satisfy:

(i) $\tau_{1}$ and $\tau_{2}$ end at the same vertex, say $\rho_{1}$;

(ii) $\left|\sigma_{1}\right|=\left|\sigma_{2}\right|$;

(iii) $\sigma_{2}$ and $\sigma_{3}$ end at the same vertex, say $\pi_{2}$; and

(iv) $\left|\tau_{2}\right|=\left|\tau_{3}\right|$.

Then,

$$
A\left(\sigma_{1}, \tau_{1}, \lambda\right) A\left(\sigma_{2}, \tau_{2}, \lambda\right)^{*} A\left(\sigma_{3}, \tau_{3}, \lambda\right)=\delta_{\sigma_{1}, \sigma_{2}} \delta_{\tau_{2}, \tau_{3}} A\left(\sigma_{3}, \tau_{1}, \lambda\right) .
$$

(It should be noted that the assumptions (i)-(iv) are precisely what are needed to ensure that the matrices above are 'multipliable'.)

Proof. Suppose $\left|\sigma_{1}\right|=\left|\sigma_{2}\right|=k_{1},\left|\sigma_{3}\right|=k_{2}$, and $\left|\tau_{1}\right|=l_{1},\left|\tau_{2}\right|=\left|\tau_{3}\right|=l_{2}$; and suppose $\sigma_{1}$ and $\tau_{3}$ finish at $\pi_{1}$ and $\rho_{3}$ respectively.

First, deduce from the invariance under flips, for the connection value on 'macro-cells', that

$$
\begin{aligned}
\left(A\left(\sigma_{2}, \tau_{2}, \lambda\right)^{*}\right)_{\widetilde{\tau}}^{\widetilde{\sigma}} & =\left(\begin{array}{ccc}
* & \stackrel{\tau_{2}}{\rightarrow} & \rho_{1} \\
\sigma_{2} \downarrow & & \downarrow \widetilde{\sigma} \\
\pi_{2} & \stackrel{\widetilde{\tau}}{\rightarrow} & \lambda
\end{array}\right)^{-} \\
& =\sqrt{\frac{\mu\left(\pi_{2}\right) \mu\left(\rho_{1}\right)}{\mu(\lambda) \mu(*)}}\left(\begin{array}{ccc}
\rho_{1} & \stackrel{\tau_{2}^{o}}{\rightarrow} & * \\
\widetilde{\sigma} \downarrow & & \downarrow \sigma_{2} \\
\lambda & \stackrel{\widetilde{\tau}^{o}}{\rightarrow} & \pi_{2}
\end{array}\right)
\end{aligned}
$$

and that

$$
\begin{array}{rlccc}
A\left(\sigma_{3}, \tau_{3}, \lambda\right)_{\sigma^{\prime}}^{\widetilde{\tau}}= & * & \stackrel{\tau_{3}}{\rightarrow} & \rho_{3} \\
& \sigma_{3} \downarrow & & \downarrow \sigma^{\prime} \\
\pi_{2} & \stackrel{\widetilde{\tau}}{\rightarrow} & \lambda \\
\lambda & \stackrel{\widetilde{\tau}^{o}}{\rightarrow} & \pi_{2} \\
& = & \sigma^{\prime o} \downarrow & & \downarrow \sigma_{3}^{o} . \\
\rho_{3} & \stackrel{\tau_{3}^{o}}{\longrightarrow} & *
\end{array} .
$$


Hence we see that

$$
\begin{aligned}
& \left(A\left(\sigma_{1}, \tau_{1}, \lambda\right) A\left(\sigma_{2}, \tau_{2}, \lambda\right)^{*} A\left(\sigma_{3}, \tau_{3}, \lambda\right)\right)_{\sigma^{\prime}}^{\tau^{\prime}} \\
& =\sum_{\widetilde{\sigma} \in \mathcal{P}_{k_{1}}^{V}\left(\rho_{1}, \lambda\right)} \sum_{\widetilde{\tau} \in \mathcal{P}_{l_{2}}^{H}\left(\pi_{2}, \lambda\right)} A\left(\sigma_{1}, \tau_{1}, \lambda\right)_{\widetilde{\sigma}}^{\tau^{\prime}}\left(A\left(\sigma_{2}, \tau_{2}, \lambda\right)^{*}\right)_{\widetilde{\tau}}^{\widetilde{\sigma}} A\left(\sigma_{3}, \tau_{3}, \lambda\right)_{\sigma^{\prime}}^{\widetilde{\tau}} \\
& =\sum_{\widetilde{\sigma} \in \mathcal{P}_{k_{1}}^{V}\left(\rho_{1}, \lambda\right)} \sum_{\widetilde{\tau} \in \mathcal{P}_{l_{2}}^{H}\left(\pi_{2}, \lambda\right)}\left(\begin{array}{ccc}
* & \stackrel{\tau_{1}}{\rightarrow} & \rho_{1} \\
\sigma_{1} \downarrow & & \downarrow \widetilde{\sigma} \\
\pi_{1} & \stackrel{\tau^{\prime}}{\rightarrow} & \lambda
\end{array}\right) \\
& \cdot \sqrt{\frac{\mu\left(\pi_{2}\right) \mu\left(\rho_{1}\right)}{\mu(\lambda)}}\left(\begin{array}{ccc}
\rho_{1} & \stackrel{\tau_{2}^{o}}{\rightarrow} & * \\
\widetilde{\sigma} \downarrow & & \downarrow \sigma_{2} \\
\lambda & \stackrel{\tilde{\tau}^{o}}{\rightarrow} & \pi_{2}
\end{array}\right)\left(\begin{array}{ccc}
\lambda & \stackrel{\widetilde{\tau}^{o}}{\rightarrow} & \pi_{2} \\
\sigma^{\prime o} \downarrow & & \downarrow \sigma_{3}^{o} \\
\rho_{3} & \stackrel{\tau_{3}^{o}}{\rightarrow} & *
\end{array}\right) \\
& =\sum_{\widetilde{\sigma}, \widetilde{\tau}} \sqrt{\frac{\mu\left(\pi_{2}\right) \mu\left(\rho_{1}\right)}{\mu(\lambda)}}\left(\begin{array}{rrrrll}
* & \stackrel{\tau_{1}}{\rightarrow} & \rho_{1} & \stackrel{\tau_{2}^{o}}{\rightarrow} & * \\
\sigma_{1} \downarrow & & \widetilde{\sigma} \downarrow & & \downarrow \sigma_{2} \\
\pi_{1} & \stackrel{\tau^{\prime}}{\rightarrow} & \lambda & \stackrel{\widetilde{\tau}^{o}}{\rightarrow} & \pi_{2} \\
& & \sigma^{o} & \downarrow & & \downarrow \sigma_{3}^{o} \\
& & \rho_{3} & \stackrel{\tau_{3}^{o}}{\rightarrow} & *
\end{array}\right) \\
& =\sqrt{\frac{\mu\left(\pi_{2}\right) \mu\left(\rho_{1}\right)}{\mu(\lambda)}}\left(W_{\left(\left(k_{1}, k_{2}\right),\left(l_{1}, l_{2}\right)\right),\left(\left(0, k_{1}+k_{2}\right),\left(l_{1}+l_{2}, 0\right)\right)}(*, *)\right)_{\left(\tau_{1}, \tau_{2}^{o}, \sigma_{2}, \sigma_{3}^{o}\right)}^{\left(\sigma_{1}, \tau^{\prime}, \sigma^{o}, \tau^{o}\right)},
\end{aligned}
$$

where we have used Equations (3.2) and (3.3) in the second step above, and we have used the notation of Lemma 2.3 in the last step. In the same notation, let us write $k=k_{1}+k_{2}, l=l_{1}+l_{2}$, and

$$
(\mathbf{k}, \mathbf{l})=((k),(l)),\left(\mathbf{k}^{\prime}, \mathbf{l}^{\prime}\right)=\left(\left(k_{1}, k_{2}\right),\left(l_{1}, l_{2}\right)\right),\left(\mathbf{k}^{\prime \prime}, \mathbf{l}^{\prime \prime}\right)=((0, k),(l, 0))
$$

and

$$
W_{1}=W_{(\mathbf{k}, \mathbf{l}),\left(\mathbf{k}^{\prime}, \mathbf{l}^{\prime}\right)}(*, *), W_{2}=W_{\left(\mathbf{k}^{\prime}, \mathbf{l}^{\prime}\right),\left(\mathbf{k}^{\prime \prime}, \mathbf{l}^{\prime \prime}\right)}(*, *) .
$$

Then, we may deduce from Lemma 2.3(b) and the flatness axiom (i.e., what we refer to as the $4 *$ flatness condition) that

$$
\left(W_{1} W_{2}\right)_{\left(\tau_{1}, \tau_{2}^{o}, \sigma_{2}, \sigma_{3}^{o}\right)}^{\left(\sigma_{1}, \sigma_{3}^{\prime o}, \tau^{\prime}, o\right.}=\delta_{\tau_{1}}^{\tau_{1}^{\prime}} \delta_{\tau_{2}}^{\tau_{3}} \delta_{\sigma_{2}}^{\sigma_{1}} \delta_{\sigma_{3}}^{\sigma_{3}^{\prime}}
$$

and since the $W_{i}, i=1,2$ are unitary, it follows that

$$
\left(W_{2}\right)_{\left(\tau_{1}, \tau_{2}^{o}, \sigma_{2}, \sigma_{3}^{o}\right)}^{\left(\sigma_{1}, \sigma^{\prime}\right)}=\delta_{\sigma_{2}}^{\sigma_{1}} \delta_{\tau_{3}}^{\tau_{2}}\left(\left(W_{1}\right)_{\left(\sigma_{1}, \tau^{\prime}, \sigma^{\prime}, \tau_{3}^{o}\right)}^{\left(\sigma_{1}, \tau^{o}, \tau_{1}^{o}\right)}\right)^{-}
$$


We finally conclude — from yet another application of flip-invariance that

$$
\begin{aligned}
& \delta_{\sigma_{2}}^{\sigma_{1}} \delta_{\tau_{3}}^{\tau_{2}} A\left(\sigma_{3}, \tau_{1}, \lambda\right)_{\sigma^{\prime}}^{\tau^{\prime}} \\
& =\delta_{\sigma_{2}}^{\sigma_{1}} \delta_{\tau_{3}}^{\tau_{2}}\left(\begin{array}{ccc}
* & \stackrel{\tau_{1}}{\rightarrow} & \rho_{1} \\
\sigma_{3} \downarrow & & \downarrow \sigma^{\prime} \\
\pi_{2} & \stackrel{\tau^{\prime}}{\rightarrow} & \lambda
\end{array}\right) \\
& =\delta_{\sigma_{2}}^{\sigma_{1}} \delta_{\tau_{3}}^{\tau_{2}}\left(\begin{array}{ccc}
* & \stackrel{\tau_{1}}{\rightarrow} & \rho_{3} \\
\sigma_{3} \downarrow & & \downarrow \sigma^{\prime} \\
\pi_{1} & \stackrel{\tau^{\prime}}{\rightarrow} & \lambda
\end{array}\right) \\
& =\delta_{\sigma_{2}}^{\sigma_{1}} \delta_{\tau_{3}}^{\tau_{2}} \sqrt{\frac{\mu\left(\pi_{1}\right) \mu\left(\rho_{3}\right)}{\mu(\lambda)}}\left(\begin{array}{ccc}
\pi_{1} & \stackrel{\tau^{\prime}}{\rightarrow} & \lambda \\
\sigma_{3}^{o} \downarrow & & \downarrow \sigma^{\prime o} \\
* & \stackrel{\tau_{1}}{\rightarrow} & \rho_{3}
\end{array}\right)^{-} \\
& =\sqrt{\frac{\mu\left(\pi_{1}\right) \mu\left(\rho_{3}\right)}{\mu(\lambda)}} \delta_{\sigma_{2}}^{\sigma_{1}} \delta_{\tau_{3}}^{\tau_{2}}\left(\left(W_{1}\right)_{\left(\sigma_{1}, \tau^{\prime}, \sigma^{\prime}, \tau_{3}^{o}\right)}^{\left(\sigma_{1}, \sigma^{o}, \tau_{1}^{o}\right)}\right)^{-} \\
& =\sqrt{\frac{\mu\left(\pi_{1}\right) \mu\left(\rho_{3}\right)}{\mu(\lambda)}}\left(W_{2}\right)_{\left(\tau_{1}, \tau_{2}^{o}, \sigma_{2}, \sigma_{3}^{o}\right)}^{\left(\sigma_{1}, \sigma^{\prime}\right)} \\
& =\left(A\left(\sigma_{1}, \tau_{1}, \lambda\right) A\left(\sigma_{2}, \tau_{2}, \lambda\right)^{*} A\left(\sigma_{3}, \tau_{3}, \lambda\right)\right)_{\sigma^{\prime}}^{\tau^{\prime}},
\end{aligned}
$$

as desired. (We have used Equations (3.5) and (3.4) in the last two steps of the above array of equations.)

We wish to show, among other things, that ' $4 *$ flatness', which was used in the above derivation of ' $1 *$ flatness', can also be deduced as a consequence of ' $1 *$ flatness'. So, in what follows, we assume that we are working with the modified definition of a paragroup, where we have replaced the flatness axiom by the above proposition; and we will deduce various consequences of the above ' $1 *$ flatness' condition.

In the sequel, we shall assume, unless explicitly indicated otherwise, that the symbols $*, \pi, \rho, \lambda, \sigma, \tau, k, l$ always satisfy the following relationship: There exist $i, p, r \in\{0,1\}$ such that

$$
*=*_{i}, \pi \in B_{p i}, \rho \in B_{i r}, \lambda \in B_{p r}, \sigma \in \mathcal{P}_{k}^{V}(*, \pi), \tau \in \mathcal{P}_{l}^{H}(*, \rho) .
$$

Proposition 3.3. $A(\sigma, \tau, \lambda)$ is a partial isometry (possibly equal to 0 ), whose 'inital and final projections' have the following properties:

(i) The projection $E(\sigma, \rho, \lambda)=A(\sigma, \tau, \lambda)^{*} A(\sigma, \tau, \lambda)$ is independent of the path $\tau$ (and, in particular, of its length); and

(ii) the projection $F(\pi, \tau, \lambda)=A(\sigma, \tau, \lambda) A(\sigma, \tau, \lambda)^{*}$ is independent of the path $\sigma$ (and, in particular, of its length). 
Proof. Setting $\sigma_{i}=\sigma, \tau_{i}=\tau$ for all $i$ in Equation (3.1), we see that the matrix $A(\sigma, \tau, \lambda)$ satisfies the equation $A A^{*} A=A$, which is the defining characteristic of a partial isometry.

Let $\sigma_{1}, \sigma_{3} \in \mathcal{P}^{V}(*, \pi)$ and $\tau_{1}, \tau_{2} \in \mathcal{P}^{H}(*, \rho)$ be arbitrary (with possibly different lengths); set $\sigma_{2}=\sigma_{1}$ and $\tau_{3}=\tau_{2}$, and deduce from Equation (3.1) that

range $A\left(\sigma_{3}, \tau_{1}, \lambda\right) \subset$ range $A\left(\sigma_{1}, \tau_{1}, \lambda\right)$, ker $A\left(\sigma_{3}, \tau_{1}, \lambda\right) \supset$ ker $A\left(\sigma_{3}, \tau_{3}, \lambda\right)$;

by symmetry, we see that $\operatorname{ran} A(\sigma, \tau, \lambda)$ (resp., ker $A(\sigma, \tau, \lambda))$ is independent of $\sigma$ (resp., $\tau$ ), as desired.

Proposition 3.4. (a) Suppose that $\sigma, \sigma^{\prime}$ are vertical paths of the same length starting at $*$ (whose finishing points $\pi$ and $\pi^{\prime}$ may be distinct), and that $\tau, \tau^{\prime}$ are paths of possibly different lengths between $*$ and $\rho$. Then

$$
A(\sigma, \tau, \lambda) A\left(\sigma^{\prime}, \tau^{\prime}, \lambda\right)^{*}=\delta_{\sigma, \sigma^{\prime}} V\left(\tau, \tau^{\prime}, \pi^{\prime}, \lambda\right)
$$

where $V\left(\tau, \tau^{\prime}, \pi, \lambda\right)$ is a partial isometry with initial projection $F\left(\pi, \tau^{\prime}\right.$, $\lambda)$ and final projection $F(\pi, \tau, \lambda)$.

(b) Dually, if $\sigma, \sigma^{\prime} \in \mathcal{P}^{V}(*, \pi)$, if $\tau \in \mathcal{P}^{H}(*, \rho), \tau^{\prime} \in \mathcal{P}^{H}\left(*, \rho^{\prime}\right)$ and if $|\tau|=$ $\left|\tau^{\prime}\right|$, then

$$
A(\sigma, \tau, \lambda)^{*} A\left(\sigma^{\prime}, \tau^{\prime}, \lambda\right)=\delta_{\tau, \tau^{\prime}} U\left(\sigma, \sigma^{\prime}, \rho^{\prime}, \lambda\right),
$$

where $U\left(\sigma, \sigma^{\prime}, \rho^{\prime}, \lambda\right)$ is a partial isometry with initial projection $E\left(\sigma^{\prime}, \rho\right.$, $\lambda)$ and final projection $E(\sigma, \rho, \lambda)$.

Proof. (a) Note that the matrix products make sense under the hypotheses, and that the fact that $A\left(\sigma^{\prime}, \tau^{\prime}, \lambda\right)^{*}$ is a partial isometry, coupled with Equation (3.1), shows that

$$
\begin{aligned}
A(\sigma, \tau, \lambda) A\left(\sigma^{\prime}, \tau^{\prime}, \lambda\right)^{*} & =A(\sigma, \tau, \lambda) A\left(\sigma^{\prime}, \tau^{\prime}, \lambda\right)^{*} A\left(\sigma^{\prime}, \tau^{\prime}, \lambda\right) A\left(\sigma^{\prime}, \tau^{\prime}, \lambda\right)^{*} \\
& =\delta_{\sigma, \sigma^{\prime}} A\left(\sigma^{\prime}, \tau, \lambda\right) A\left(\sigma^{\prime}, \tau^{\prime}, \lambda\right)^{*}
\end{aligned}
$$

and the desired conclusion follows from the following observation (which applies in this case): If $V, V^{\prime}$ are partial isometries with the same initial space, then $V\left(V^{\prime}\right)^{*}$ is a partial isometry whose initial (resp., final) projection is the same as the final projection of $V^{\prime}$ (resp., $V$ ).

The proof of (b) is identical.

Proposition 3.5. (a) If $\tau$ and $\tau^{\prime}$ are distinct horizontal paths of the same length starting at $*$ (with possibly different end-points), then, for any vertex $\pi$, the projections $F(\pi, \tau, \lambda)$ and $F\left(\pi, \tau^{\prime}, \lambda\right)$ are mutually orthogonal.

(b) If $\sigma$ and $\sigma^{\prime}$ are distinct vertical paths of the same length starting at * (with possibly different end-points), then, for any vertex $\rho$, the projections $E(\sigma, \rho, \lambda)$ and $E\left(\sigma^{\prime}, \rho, \lambda\right)$ are mutually orthogonal. 
Proof. (a)

$$
\begin{aligned}
F(\pi, \tau, \lambda) F\left(\pi, \tau^{\prime}, \lambda\right) & =A(\sigma, \tau, \lambda) A(\sigma, \tau, \lambda)^{*} A\left(\sigma, \tau^{\prime}, \lambda\right) A\left(\sigma, \tau^{\prime}, \lambda\right)^{*} \\
& =\delta_{\tau \tau^{\prime}} A(\sigma, \tau, \lambda) A\left(\sigma, \tau^{\prime}, \lambda\right)^{*} \\
& =0
\end{aligned}
$$

The proof of (b) is similar.

\section{Proposition 3.6. (a)}

$$
\sum_{\tau \in \mathcal{P}_{l}^{H}(*,-)} F(\pi, \tau, \lambda)=I_{\mathcal{P}_{l}^{H}(\pi, \lambda)},
$$

where we write $I_{X}$ to denote the identity matrix with rows and columns indexed by the set $X$.

(b)

$$
\sum_{\sigma \in \mathcal{P}_{k}^{V}(*,-)} E(\sigma, \rho, \lambda)=I_{\mathcal{P}_{k}^{V}(\rho, \lambda)} .
$$

Proof. (a) Arbitrarily choose and fix some $\sigma \in \mathcal{P}_{k}^{V}(*, \pi)$. Then, by the unitarity axiom in a paragroup, we have, for each $\tau^{\prime} \in \mathcal{P}_{l}^{H}(\pi, \lambda)$,

$$
1=\sum_{\tau, \sigma^{\prime}}\left|\begin{array}{ccc}
* & \stackrel{\tau}{\rightarrow} & \cdot \\
\sigma & & \downarrow \sigma^{\prime} \\
\pi & \stackrel{\tau^{\prime}}{\rightarrow} & \lambda
\end{array}\right|^{2} .
$$

Summing over $\tau^{\prime} \in \mathcal{P}_{l}^{H}(\pi, \lambda)$, we find that

$$
\begin{aligned}
\left|\mathcal{P}_{l}^{H}(\pi, \lambda)\right| & =\sum_{\tau^{\prime} \in \mathcal{P}_{l}^{H}(\pi, \lambda)} \sum_{\tau, \sigma^{\prime}}\left|\begin{array}{ccc}
\sigma \downarrow & \stackrel{\tau}{\rightarrow} & \downarrow \\
\pi & \stackrel{\tau^{\prime}}{\rightarrow} & \lambda
\end{array}\right|^{2} \\
& =\sum_{\tau^{\prime} \in \mathcal{P}_{l}^{H}(\pi, \lambda)} \sum_{\tau, \sigma^{\prime}}\left|A(\sigma, \tau, \lambda)_{\sigma^{\prime}}^{\tau^{\prime}}\right|^{2} \\
& =\sum_{\tau \in \mathcal{P}_{l}^{H}(*,-)}\|A(\sigma, \tau, \lambda)\|_{2}^{2} \\
& =\sum_{\tau \in \mathcal{P}_{l}^{H}(*,-)} \operatorname{Tr} F(\pi, \tau, \lambda) .
\end{aligned}
$$

(Above, and in the sequel, we use the symbols $\|\cdot\|_{2}$ and ' $\mathrm{Tr}$ ' to denote the Hilbert-Schmidt norm and the usual trace on any matrix algebra.)

Since, by Proposition 3.5, $\left\{F(\pi, \tau, \lambda): \tau \in \mathcal{P}_{l}^{H}(*,-)\right\}$ is a collection of mutually orthogonal projections in $\operatorname{Mat}_{\mathcal{P}_{l}^{H}(\pi, \lambda)}(\mathbb{C})$, the desired conclusion follows.

The proof of (b) is similar. 
Remark 3.7. On the one hand,

$$
\|A(\sigma, \tau, \lambda)\|_{2}^{2}=\operatorname{Tr}\left(A(\sigma, \tau, \lambda) A(\sigma, \tau, \lambda)^{*}\right)=\operatorname{Tr}(F(\pi, \tau, \lambda))
$$

is independent of $\sigma$; on the other,

$$
\|A(\sigma, \tau, \lambda)\|_{2}^{2}=\operatorname{Tr}\left(A(\sigma, \tau, \lambda)^{*} A(\sigma, \tau, \lambda)\right)=\operatorname{Tr}(E(\sigma, \rho, \lambda))
$$

is independent of $\tau$. Hence we see that $\|A(\sigma, \tau, \lambda)\|^{2}$ is independent of $\sigma$ and $\tau$ (and hence of $k$ and $l$ ), and depends only on $\pi, \rho, \lambda$. We define

$$
f(\pi, \rho, \lambda)=\|A(\sigma, \tau, \lambda)\|_{2}^{2} .
$$

Lemma 3.8. Let $i, p, r \in\{0,1\}$ be arbitrary.

(a) For any $\pi \in B_{p i}$ and $\rho \in B_{\text {ir }}$, there exists at least one $\lambda \in B_{p r}$ such that $f(\pi, \rho, \lambda)>0$.

(b) For any $\pi \in B_{p i}$ and $\lambda \in B_{p r}$, there exists at least one $\rho \in B_{\text {ir }}$ such that $f(\pi, \rho, \lambda)>0$.

Proof. (a) Choose some $\sigma \in \mathcal{P}^{V}(*, \pi), \tau \in \mathcal{P}^{H}(*, \rho)$. The unitarity axiom implies the existence of a $\lambda$ and $\sigma^{\prime} \in \mathcal{P}_{|\sigma|}^{V}(\rho, \lambda), \tau^{\prime} \in \mathcal{P}_{|\tau|}^{H}(\pi, \lambda)$ such that

$$
\begin{array}{rll}
\pi & \stackrel{\tau^{\prime}}{\rightarrow} & \lambda \\
\sigma^{o} \downarrow & & \downarrow \sigma^{\prime o} \neq 0 . \\
* & \stackrel{\tau}{\rightarrow} & \rho
\end{array}
$$

Then, by flip-invariance, we see that

$$
\begin{aligned}
A(\sigma, \tau, \lambda)_{\sigma^{\prime}}^{\tau^{\prime}} & =\begin{array}{rlll} 
& * & \stackrel{\tau}{\rightarrow} & \rho \\
& \pi & \stackrel{\tau^{\prime}}{\rightarrow} & \lambda
\end{array} \\
& =\sqrt{\frac{\mu(\pi) \mu(\rho)}{\mu(\lambda)}}\left(\begin{array}{rrrr}
\pi & \stackrel{\tau^{\prime}}{\rightarrow} & \lambda \\
\sigma^{o} \downarrow & & \downarrow \sigma^{\prime o} \\
* & \stackrel{\tau}{\rightarrow} & \rho
\end{array}\right){ }^{-} \\
& \neq 0 .
\end{aligned}
$$

The proof of (b) is similar.

\section{Proposition 3.9.}

(a)

$$
\begin{array}{rll}
* & \stackrel{\tau}{\rightarrow} & * \\
& \downarrow & \\
* & \stackrel{\tau^{\prime}}{\rightarrow} & *
\end{array}
$$

(b) For any $\pi \in B_{\text {pi }}$ and $\rho \in B_{\text {ir }}$,

$$
\sum_{\lambda \in B_{p r}} f(\pi, \rho, \lambda) \mu(\lambda)=\mu(\pi) \mu(\rho) .
$$


Proof. First of all, note that we must have

$$
|\sigma|=\left|\sigma^{\prime}\right|=2 m,|\tau|=\left|\tau^{\prime}\right|=2 n
$$

for some integers $m, n$. So we may write $\sigma=\sigma_{1} \sigma_{2}, \sigma^{\prime}=\sigma_{1}^{\prime} \sigma_{2}^{\prime}$ and $\tau=$ $\tau_{1} \tau_{2}, \tau^{\prime}=\tau_{1}^{\prime} \tau_{2}^{\prime}$, where $\left|\sigma_{i}\right|=\left|\sigma_{i}^{\prime}\right|=m,\left|\tau_{i}\right|=\left|\tau_{i}^{\prime}\right|=n$, for $i=1,2$. (Here and elsewhere, we shall denote concatenation of paths by merely juxtaposing the symbols; thus, the symbol $\sigma_{1} \sigma_{2}$ denotes the path obtained by first traversing the path $\sigma_{1}$ and then going on to traverse the path $\sigma_{2}$; this naturally demands that $\sigma_{1}$ finishes where $\sigma_{2}$ starts.)

Suppose that $\sigma_{1}$ and $\sigma_{1}^{\prime}$ finish at $\pi$ and $\pi^{\prime}$ respectively, and that $\tau_{1}$ and $\tau_{1}^{\prime}$ finish at $\rho$ and $\rho^{\prime}$ respectively. Now, compute thus:

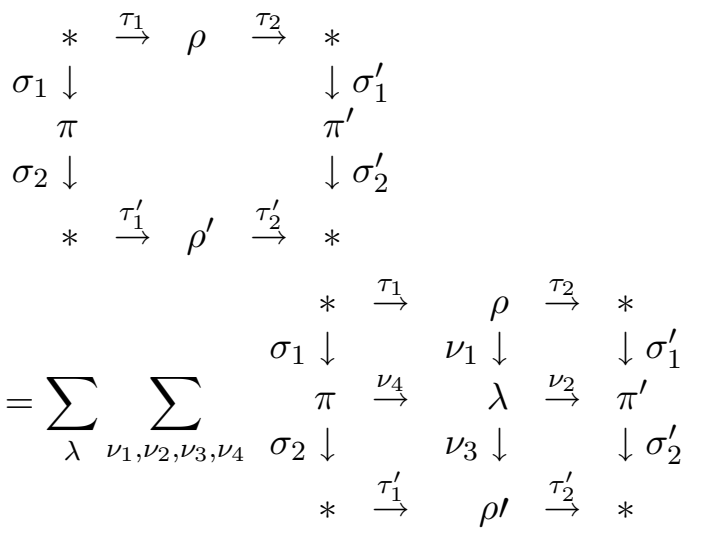

$=\sum_{\lambda} \sum_{\nu_{1}, \nu_{2}, \nu_{3}, \nu_{4}} A\left(\sigma_{1}, \tau_{1}, \lambda\right)_{\nu_{1}}^{\nu_{4}} \sqrt{\frac{\mu(\lambda)}{\mu(\rho) \mu\left(\pi^{\prime}\right)}}\left(A\left(\sigma_{1}^{\prime}, \tau_{2}^{o}, \lambda\right)_{\nu_{1}}^{\nu_{2}^{o}}\right)^{-}$

$\cdot A\left(\sigma_{2}^{\prime o}, \tau_{2}^{\prime o}, \lambda\right)_{\nu_{3}^{o}}^{\nu_{0}^{o}} \sqrt{\frac{\mu(\lambda)}{\mu\left(\rho^{\prime}\right) \mu(\pi)}}\left(A\left(\sigma_{2}^{o}, \tau_{1}^{\prime}, \lambda\right)_{\nu_{3}^{o}}^{\nu_{4}}\right)^{-}$

$=\sum_{\lambda} \frac{\mu(\lambda)}{\sqrt{\mu(\pi) \mu\left(\pi^{\prime}\right) \mu(\rho) \mu\left(\rho^{\prime}\right)}} \times$

- $\operatorname{Tr}\left(A\left(\sigma_{1}, \tau_{1}, \lambda\right) A\left(\sigma_{1}^{\prime}, \tau_{2}^{o}, \lambda\right)^{*} A\left(\sigma_{2}^{\prime o}, \tau_{2}^{\prime o}, \lambda\right) A\left(\sigma_{2}^{o}, \tau_{1}^{\prime}, \lambda\right)^{*}\right)$

$=\sum_{\lambda} \frac{\mu(\lambda)}{\sqrt{\mu(\pi) \mu\left(\pi^{\prime}\right) \mu(\rho) \mu\left(\rho^{\prime}\right)}} \delta_{\sigma_{1}, \sigma_{1}^{\prime}} \delta_{\tau_{2}, \tau_{2}^{\prime}} \operatorname{Tr}\left(A\left(\sigma_{2}^{\prime o}, \tau_{1}, \lambda\right) A\left(\sigma_{2}^{o}, \tau_{1}^{\prime}, \lambda\right)^{*}\right)$

$(*)=\sum_{\lambda} \frac{\mu(\lambda)}{\sqrt{\mu(\pi) \mu\left(\pi^{\prime}\right) \mu(\rho) \mu\left(\rho^{\prime}\right)}} \delta_{\sigma_{1}, \sigma_{1}^{\prime}} \delta_{\tau_{2}, \tau_{2}^{\prime}} \delta_{\sigma_{2}, \sigma_{2}^{\prime}} \operatorname{Tr} V\left(\tau_{1}, \tau_{1}^{\prime}, \pi, \lambda\right)$

$(* *)=\sum_{\lambda} \frac{\mu(\lambda)}{\sqrt{\mu(\pi) \mu\left(\pi^{\prime}\right) \mu(\rho) \mu\left(\rho^{\prime}\right)}} \delta_{\sigma_{1}, \sigma_{1}^{\prime}} \delta_{\tau_{2}, \tau_{2}^{\prime}} \delta_{\sigma_{2}, \sigma_{2}^{\prime}} \delta_{\tau_{1}, \tau_{1}^{\prime}} \operatorname{Tr} F\left(\tau_{1}, \pi, \lambda\right)$

$=\delta_{\sigma, \sigma^{\prime}} \delta_{\tau, \tau^{\prime}} K(\pi, \rho)$, 
where we have used: (a) Proposition 3.4 in step (*) (and used the two Kronecker deltas to ensure that the hypotheses of that proposition are indeed satisfied), (b) Proposition 3.5 (a) and the fact that a partial isometry with mutually orthogonal initial and final spaces must necessarily have 0 trace, in step $(* *)$ and $(\mathrm{c})$ the notation

$$
K(\pi, \rho)=\sum_{\lambda} \frac{\mu(\lambda)}{\mu(\pi) \mu(\rho)} f(\pi, \rho, \lambda)
$$

in the last step.

It is clear that $K(\pi, \rho) \geq 0$; but, in fact, it follows from Lemma 3.8 that $K(\pi, \rho)>0$. It follows, therefore, that $A(\sigma, \tau, *)$ is a partial isometry which has the positive entry $K(\pi, \rho)$ in the $(\tau, \sigma)$ entry and has all other entries equal to 0 ; hence we see that

$$
K(\pi, \rho)=\|A(\sigma, \tau, *)\|=1
$$

(since the norm of a partial isometry is 0 or 1 , and we know that $K(\pi, \rho)>$ $0)$.

The truth of both parts of the proposition is seen to follow.

\section{Derivation of the formula.}

Suppose one starts with a 'finite-depth hyperfinite subfactor' $R_{0} \subset R_{1}$; then for the associated paragroup, the set $B_{i j}$ can be identified with the collection of (isomorphism classes of) irreducible $R_{i}-R_{j}$ bimodules that 'occur in the tower of the basic construction' - see [AO1] or [VJ]. In this case, we have $*_{i}={ }_{R_{i}} L^{2}\left(R_{i}\right)_{R_{i}}$ and $\alpha_{i, 1-i}={ }_{R_{i}} L^{2}\left(R_{1}\right)_{R_{1-i}}$ for $i=0,1$. Further, it is true that if $X_{i j}$ is such a bimodule, then, we have

$$
\mu\left(X_{i j}\right)=\sqrt{\operatorname{dim}\left(R_{i} X\right) \operatorname{dim}\left(X_{R_{j}}\right)},
$$

where $\operatorname{dim}\left({ }_{P} X\right)$ (resp., $\left.\operatorname{dim}\left(X_{P}\right)\right)$ denotes the $P$-dimension of the left (resp., right) $P$-module $X$.

A vertical edge starting at $\rho \in B_{i r}$ and finishing at $\gamma \in B_{1-i, r}$ corresponds to a member of a maximal family (chosen and fixed once and for all) of $R_{1-i}-R_{r}$ linear coisometries from $\alpha_{1-i, i} \otimes_{R_{i}} \rho$ to $\gamma$; while the same edge, when thought of as starting from $\gamma$ and finishing at $\rho$ is identified with the image, under a suitable ('left flip') Frobenius reciprocity map (cf. [EK]), of the intertwiner corresponding to the 'unreversed edge'. Similar comments apply to horizontal graphs, which correspond to tensoring on the right, just as the vertical graphs correspond to tensoring on the left. (Making a different choice of the 'maximal sets of co-isometric intertwiners' corresponds exactly to replacing the connection by an equivalent one.)

The point we wish to make here is that $f(\pi, \rho, \lambda)$ is now nothing but the fusion coefficient $\left\langle\pi \otimes_{R_{i}} \rho, \lambda\right\rangle$. 
Theorem 4.1. With the foregoing notation, if $\pi_{0} \in B_{p i}, \rho_{0} \in B_{i r}$ and $\lambda_{0} \in$ $B_{p r}$, then $f\left(\pi_{0}, \rho_{0}, \lambda_{0}\right)$ is nothing but the the fusion coefficient $\left\langle\pi_{0} \otimes_{R_{i}} \rho_{0}, \lambda_{0}\right\rangle$, i.e., the 'multiplicity with which the bimodule $\lambda_{0}$ features in $\pi_{0} \otimes_{R_{i}} \rho_{0}$ '.

Proof. We shall ignore the 'tensor-product' symbol, and simply write such symbols as $\left(\alpha_{01} \alpha_{10}\right)^{m}$ to denote appropriate tensor-products; thus, for instance,

$$
\left(\alpha_{01} \alpha_{10}\right)^{2}=\alpha_{01} \otimes_{R_{1}} \alpha_{10} \otimes_{R_{0}} \alpha_{01} \otimes_{R_{1}} \alpha_{10}
$$

Let us write

$$
X_{k}= \begin{cases}\left(\alpha_{i, 1-i} \alpha_{1-i, i}\right)^{m} & \text { if } k=2 m \\ \alpha_{1-i, i}\left(\alpha_{i, 1-i} \alpha_{1-i, i}\right)^{m} & \text { if } k=2 m+1\end{cases}
$$

and

$$
Y_{l}=\left\{\begin{array}{ll}
\left(\alpha_{i, 1-i} \alpha_{1-i, i}\right)^{m} & \text { if } l=2 m \\
\alpha_{i, 1-i}\left(\alpha_{i, 1-i} \alpha_{1-i, i}\right)^{m} & \text { if } l=2 m+1
\end{array} .\right.
$$

Fix a positive integer $k$ (resp., $l$ ) such that: (i) $\mathcal{P}_{k}^{V}\left(*_{i}, \pi_{0}\right) \neq \emptyset$ (resp., $\mathcal{P}_{l}^{H}\left(*_{i}, \rho_{0}\right) \neq \emptyset$ ), and (ii) $k$ (resp., $l$ ) is at least as large as the distance, in $V_{i}$ (resp., $\left.H_{i}\right)$ from $*_{i}$ to any vertex in $B_{p i}$ (resp., $B_{i r}$ ). It then follows that $\mathcal{P}_{k}^{V}\left(*_{i}, \pi\right) \neq \emptyset \forall \pi \in B_{p i}$ (resp., $\left.\mathcal{P}_{l}^{H}\left(*_{i}, \rho\right) \neq \emptyset \forall \rho \in B_{i r}\right)$.

Note that $X_{k}$ is an $R_{p}-R_{i}$ bimodule and $Y_{l}$ is an $R_{i}-R_{r}$ bimodule. For the sake of typographical convenience, let us write

$$
\begin{aligned}
A_{00} & ={\text { End } R_{i}}\left(L^{2}\left(R_{i}\right)\right)_{R_{i}}=\mathbb{C}, \\
A_{k 0} & =\text { End } R_{p}\left(X_{k}\right) R_{i}, \\
A_{0 l} & =\text { End } R_{i}\left(Y_{l}\right)_{R_{r}}, \\
A_{k l} & ={\text { End } R_{p}}\left(X_{k} \otimes_{R_{i}} Y_{l}\right)_{R_{r}} .
\end{aligned}
$$

We will be interested in the square

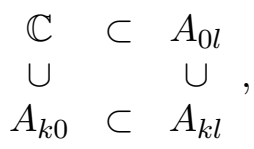

where the inclusion map in the second row (resp., column) is given by $S \mapsto$ $S \otimes_{R_{i}} \operatorname{id}_{Y_{l}}$ (resp., $T \mapsto \operatorname{id}_{X_{k}} \otimes_{R_{i}} T$ ).

It will be necessary to use the 'path-model' — see [JS] for instance - for the above algebras; for this, we shall consider the admissible tuples given by $(\mathbf{k}, \mathbf{l})=((k),(l))$ and $\left(\mathbf{k}^{\prime}, \mathbf{l}^{\prime}\right)=((0, k),(l, 0))$, and we will find it convenient to use the notation $\mathcal{P}_{k l}^{V H}\left(*_{i}, \lambda\right)$ (resp., $\mathcal{P}_{l k}^{H V}\left(*_{i}, \lambda\right)$ ) to denote the set of all (south-west) paths of type $(\mathbf{k}, \mathbf{l})$ (resp., (north-east) paths of type $\left.\left(\mathbf{k}^{\prime}, \mathbf{l}^{\prime}\right)\right)$ from $*_{i}$ to $\lambda$. We shall also consistently use the notation $f(\xi)$ to denote the vertex where a path $\xi$ finishes. When we want to consider paths 
with possibly differing finishing points, we shall also use the (abbreviated) symbols

$$
\mathcal{P}^{V H}=\coprod_{\lambda \in B_{p r}} \mathcal{P}_{k l}^{V H}\left(*_{i}, \lambda\right), \mathcal{P}^{H V}=\coprod_{\lambda \in B_{p r}} \mathcal{P}_{l k}^{H V}\left(*_{i}, \lambda\right) .
$$

It is then true - see [JS ], for instance - that we can find two subsets $\mathcal{B}_{k l}$ and $\mathcal{B}_{l k}$ of $A_{k l}$, given by:

$$
\mathcal{B}_{k l}=\left\{\left(\xi^{+}, \xi^{-}\right): \xi^{ \pm} \in \mathcal{P}^{V H}, \text { and } f\left(\xi^{+}\right)=f\left(\xi^{-}\right)\right\}
$$

and

$$
\mathcal{B}_{l k}=\left\{\left(\eta^{+}, \eta^{-}\right): \eta^{ \pm} \in \mathcal{P}^{H V}, \text { and } f\left(\eta^{+}\right)=f\left(\eta^{-}\right)\right\},
$$

which have the following properties:

(a) Both $\mathcal{B}_{k l}$ and $\mathcal{B}_{l k}$ are systems of 'generalised matrix units for $A_{k l}$ ', meaning:

(i) They are both (vector space) bases for $A_{k l}$;

(ii) $\left(\xi^{+}, \xi^{-}\right)^{*}=\left(\xi^{-}, \xi^{+}\right)$and $\left(\xi_{1}^{+}, \xi_{1}^{-}\right) \cdot\left(\xi_{2}^{+}, \xi_{2}^{-}\right)=\delta_{\xi_{1}^{-}, \xi_{2}^{+}}\left(\xi_{1}^{+}, \xi_{2}^{-}\right)$;

(iii) $\left(\eta^{+}, \eta^{-}\right)^{*}=\left(\eta^{-}, \eta^{+}\right)$and $\left(\eta_{1}^{+}, \eta_{1}^{-}\right) \cdot\left(\eta_{2}^{+}, \eta_{2}^{-}\right)=\delta_{\eta_{1}^{-}, \eta_{2}^{+}}\left(\eta_{1}^{+}, \eta_{2}^{-}\right)$.

(b) The basis $\mathcal{B}_{k l}$ is well-behaved with respect to the tower $A_{00} \subset A_{k 0} \subset$ $A_{k l}$ in the following sense: If $\sigma^{ \pm} \in \mathcal{P}_{k}^{V}\left(*_{i},-\right)$ satisfy $f\left(\sigma^{+}\right)=f\left(\sigma^{-}\right)$, then $\left(\sigma^{+} \tau, \sigma^{-} \tau\right) \in \mathcal{B}_{k l}$ whenever $\tau \in \mathcal{P}_{l}^{H}\left(f\left(\sigma^{+}\right),-\right)$; in fact, if we define

$$
\left(\sigma^{+}, \sigma^{-}\right)=\sum_{\tau \in \mathcal{P}_{l}^{H}\left(f\left(\sigma^{+}\right),-\right)}\left(\sigma^{+} \tau, \sigma^{-} \tau\right)
$$

and

$$
\mathcal{B}_{k 0}=\left\{\left(\sigma^{+}, \sigma^{-}\right): \sigma^{ \pm} \in \mathcal{P}_{k}^{V}\left(*_{i},-\right) \text { and } f\left(\sigma^{+}\right)=f\left(\sigma^{-}\right)\right\},
$$

then $\mathcal{B}_{k 0}$ is a 'system of generalised matrix units for $A_{k 0}$ '.

(c) The basis $\mathcal{B}_{l k}$ is well-behaved with respect to the tower $A_{00} \subset A_{0 l} \subset$ $A_{k l}$ in the following sense: If $\tau^{ \pm} \in \mathcal{P}_{l}^{H}\left(*_{i},-\right)$ satisfy $f\left(\tau^{+}\right)=f\left(\tau^{-}\right)$, then $\left(\tau^{+} \sigma, \tau^{-} \sigma\right) \in \mathcal{B}_{l k}$ whenever $\sigma \in \mathcal{P}_{k}^{V}\left(f\left(\tau^{+}\right),-\right)$; in fact, if we define

$$
\left(\tau^{+}, \tau^{-}\right)=\sum_{\sigma \in \mathcal{P}_{k}^{V}\left(f\left(\tau^{+}\right),-\right)}\left(\tau^{+} \sigma, \tau^{-} \sigma\right)
$$

and

$$
\mathcal{B}_{0 l}=\left\{\left(\tau^{+}, \tau^{-}\right): \tau^{ \pm} \in \mathcal{P}_{l}^{H}\left(*_{i},-\right) \text { and } f\left(\tau^{+}\right)=f\left(\tau^{-}\right)\right\},
$$

then $\mathcal{B}_{0 l}$ is a 'system of generalised matrix units for $A_{0 l}$ '.

(d) The two bases are related via the connection, as follows:

$$
\left(\xi^{+}, \xi^{-}\right)=\sum_{\eta^{ \pm} \in \mathcal{P}_{l k}^{H V}\left(*_{i}, f\left(\xi^{+}\right)\right)} W_{\eta^{+}}^{\xi^{+}} \overline{W_{\eta^{-}}^{\xi^{-}}}\left(\eta^{+}, \eta^{-}\right),
$$


for all $\left(\xi^{+}, \xi^{-}\right) \in \mathcal{B}_{k l}$.

It is seen from the above statements regarding 'generalised matrix units' that a basis vector $\left(\xi^{+}, \xi^{-}\right) \in \mathcal{B}_{k l}$ is a projection if and only if $\xi^{+}=\xi^{-}$; and that, further, if we define $p_{k 0}(\pi)$ to be the sum of all the projections in $\mathcal{B}_{k 0}$ which 'finish' at $\pi$, then $\left\{p_{k 0}(\pi): \pi \in B_{p i}\right\}$ is the set of (all the) minimal central projections of $A_{k 0}$. In fact, $p_{k 0}(\pi)$ is nothing but the projection onto the isotypical sub-bimodule of $X_{k}$ of type $\pi$. It should be clear that analogous statements, with $(\pi, k 0)$ replaced by $(\rho, 0 l)$ and $(\lambda, k l)$, are valid.

Now let us fix a $\sigma_{0} \in \mathcal{P}_{k}^{V}\left(*_{i}, \pi_{0}\right)$ and a $\tau_{0} \in \mathcal{P}_{l}^{H}\left(*_{i}, \rho_{0}\right)$, and let us write $p_{k 0}^{0}=\left(\sigma_{0}, \sigma_{0}\right)\left(\in \mathcal{B}_{k 0}\right)$ and $p_{0 l}^{0}=\left(\tau_{0}, \tau_{0}\right)\left(\in \mathcal{B}_{0 l}\right)$. The facts stated above, concerning 'generalised matrix units', imply that $p_{k 0}^{0}$ (resp., $p_{0 l}^{0}$ ) is a minimal projection in $A_{k 0} p_{k 0}\left(\pi_{0}\right)$ (resp., $A_{0 l} p_{0 l}\left(\rho_{0}\right)$ ), and hence that ran $p_{k 0}^{0}$ (resp., $\operatorname{ran} p_{0 l}^{0}$ ) is a model of the irreducible bimodule of type $\pi_{0}$ (resp., $\rho_{0}$ ).

The definitions, and a moment's thought reveal that the fusion coefficient $\left\langle\pi_{0} \otimes_{R_{i}} \rho_{0}, \lambda_{0}\right\rangle$ is exactly that number $N$ such that $\left(p_{k 0}^{0} p_{0 l}^{0}\right) p_{k l}\left(\lambda_{0}\right)$ is a sum of $N$ minimal projections in $A_{k l} p_{k l}\left(\lambda_{0}\right)$. Hence, it follows that if $\phi$ denotes any faithful positive tracial functional on $A_{k l}$, then

$$
\left\langle\pi_{0} \otimes_{R_{i}} \rho_{0}, \lambda_{0}\right\rangle=\frac{\phi\left(p_{k 0}^{0} p_{0 l}^{0} p_{k l}\left(\lambda_{0}\right)\right)}{\phi(q)},
$$

for any minimal projection $q \in A_{k l} p_{k l}\left(\lambda_{0}\right)$.

However, if 'tr' denotes the so-called Markov trace on $A_{k l}$, then there exists a constant $C>0$ such that if $\phi=C \operatorname{tr}$, then $\phi$ is a faithful positive tracial functional on $A_{k l}$, and further,

$$
\phi\left(\left(\eta^{+}, \eta^{-}\right)\right)=\delta_{\eta^{+}, \eta^{-}} \mu\left(f\left(\eta^{+}\right)\right), \forall\left(\eta^{+}, \eta^{-}\right) \in \mathcal{B}_{l k} .
$$

Now deduce from the definitions and from Equation (4.2) that

$$
\begin{aligned}
& \phi\left(p_{k 0}^{0} p_{0 l}^{0} p_{k l}\left(\lambda_{0}\right)\right) \\
&= \sum_{\tau \in \mathcal{P}_{l}^{H}\left(\pi_{0}, \lambda_{0}\right)} \sum_{\sigma \in \mathcal{P}_{k}^{V}\left(\rho_{0}, \lambda_{0}\right)} \phi\left(\left(\sigma_{0} \tau, \sigma_{0} \tau\right)\left(\tau_{0} \sigma, \tau_{0} \sigma\right) p_{k l}\left(\lambda_{0}\right)\right) \\
&= \sum_{\tau \in \mathcal{P}_{l}^{H}\left(\pi_{0}, \lambda_{0}\right)} \sum_{\sigma \in \mathcal{P}_{k}^{V}\left(\rho_{0}, \lambda_{0}\right)} \sum_{\eta^{ \pm} \in \mathcal{P}_{l k}^{H V}\left(*_{i}, \lambda_{0}\right)} \phi\left(W_{\eta^{+}}^{\sigma_{0} \tau} \overline{W_{\eta^{-}}^{\sigma_{0} \tau}}\left(\eta^{+}, \eta^{-}\right)\left(\tau_{0} \sigma, \tau_{0} \sigma\right)\right) \\
&= \sum_{\tau \in \mathcal{P}_{l}^{H}\left(\pi_{0}, \lambda_{0}\right)} \sum_{\sigma \in \mathcal{P}_{k}^{V}\left(\rho_{0}, \lambda_{0}\right)} \sum_{\eta^{+} \in \mathcal{P}_{l k}^{H V}\left(*_{i}, \lambda_{0}\right)} W_{\eta^{+}}^{\sigma_{0} \tau} \overline{W_{\tau_{0} \sigma}^{\sigma_{0} \tau}} \phi\left(\left(\eta^{+}, \tau_{0} \sigma\right)\right) \\
&= \sum_{\tau \in \mathcal{P}_{l}^{H}\left(\pi_{0}, \lambda_{0}\right)} \sum_{\sigma \in \mathcal{P}_{k}^{V}\left(\rho_{0}, \lambda_{0}\right)} \sum_{\eta^{+} \in \mathcal{P}_{l k}^{H V}\left(*_{i}, \lambda_{0}\right)} W_{\eta^{+}}^{\sigma_{0} \tau} \overline{W_{\tau_{0} \sigma}^{\sigma_{0} \tau}} \delta_{\eta^{+}, \tau_{0} \sigma} \mu\left(\lambda_{0}\right) \\
& \sum_{\tau \in \mathcal{P}_{l}^{H}\left(\pi_{0}, \lambda_{0}\right)} \sum_{\sigma \in \mathcal{P}_{k}^{V}\left(\rho_{0}, \lambda_{0}\right)} W_{\tau_{0} \sigma}^{\sigma_{0} \tau} \overline{W_{\tau_{0} \sigma}^{\sigma_{0} \tau}}
\end{aligned}
$$




$$
\begin{aligned}
& =\mu\left(\lambda_{0}\right) \sum_{\tau \in \mathcal{P}_{l}^{H}\left(\pi_{0}, \lambda_{0}\right)} \sum_{\sigma \in \mathcal{P}_{k}^{V}\left(\rho_{0}, \lambda_{0}\right)}\left|W_{\tau_{0} \sigma}^{\sigma_{0} \tau}\right|^{2} \\
& =\mu\left(\lambda_{0}\right) \sum_{\tau \in \mathcal{P}_{l}^{H}\left(\pi_{0}, \lambda_{0}\right)} \sum_{\sigma \in \mathcal{P}_{k}^{V}\left(\rho_{0}, \lambda_{0}\right)}\left|A\left(\sigma_{0}, \tau_{0}, \lambda_{0}\right)_{\sigma}^{\tau}\right|^{2} \\
& =\mu\left(\lambda_{0}\right)\left\|A\left(\sigma_{0}, \tau_{0}, \lambda_{0}\right)\right\|_{2}^{2} ;
\end{aligned}
$$

and hence, by Equations (4.3) and (4.4), we see that if $\eta \in \mathcal{P}_{l k}^{H V}\left(*_{i}, \lambda_{0}\right)$, then

$$
\begin{aligned}
\left\langle\pi_{0} \otimes_{R_{i}} \rho_{0}, \lambda_{0}\right\rangle & =\frac{\phi\left(p_{k 0}^{0} p_{0 l}^{0} p_{k l}\left(\lambda_{0}\right)\right)}{\phi((\eta, \eta))} \\
& =\frac{\mu\left(\lambda_{0}\right) f\left(\pi_{0}, \rho_{0}, \lambda_{0}\right)}{\mu\left(\lambda_{0}\right)} \\
& =f\left(\pi_{0}, \rho_{0}, \lambda_{0}\right),
\end{aligned}
$$

and the proof is complete.

\section{The fusion algebras.}

We first proceed toward showing how the 'contragredient' (in the sense of bimodules) can be read off from the paragroup.

Proposition 5.1. Let $\pi \in B_{p i}, \rho \in B_{i r}, \lambda \in B_{p r}$, and let $k, l$ be positive integers such that $(p-i)-k$ and $(r-i)-l$ are even; then

$$
\begin{aligned}
\sum_{\rho^{\prime} \in B_{i r}} f\left(\pi, \rho^{\prime}, \lambda\right)\left|\mathcal{P}_{l}^{H}\left(*_{i}, \rho^{\prime}\right)\right| & =\left|\mathcal{P}_{l}^{H}(\pi, \lambda)\right| ; \\
\sum_{\pi^{\prime} \in B_{p i}} f\left(\pi^{\prime}, \rho, \lambda\right)\left|\mathcal{P}_{k}^{V}\left(*_{i}, \pi^{\prime}\right)\right| & =\left|\mathcal{P}_{k}^{V}(\rho, \lambda)\right| .
\end{aligned}
$$

Proof. Fix a $\sigma \in \mathcal{P}^{V}(*, \pi)$. Deduce from the unitarity axiom that if $\tau^{\prime} \in$ $\mathcal{P}_{l}^{H}(\pi, \lambda)$, then

$$
1=\sum_{\tau \in \mathcal{P}_{l}^{H}(*,-)} \sum_{\sigma^{\prime} \in \mathcal{P}_{k}^{V}(f(\tau), \lambda)}\left|A(\sigma, \tau, \lambda)_{\sigma^{\prime}}^{\tau^{\prime}}\right|^{2},
$$

where we have used the notation $f(\tau)$ to denote the vertex at which $\tau$ finishes. 
Now sum over $\tau^{\prime} \in \mathcal{P}_{l}^{H}(\pi, \lambda)$ to find that

$$
\begin{aligned}
\left|\mathcal{P}_{l}^{H}(\pi, \lambda)\right| & =\sum_{\tau^{\prime} \in \mathcal{P}_{l}^{H}(\pi, \lambda)} \sum_{\tau, \sigma^{\prime}}\left|A(\sigma, \tau, \lambda)_{\sigma^{\prime}}^{\tau^{\prime}}\right|^{2} \\
& =\sum_{\tau} f(\pi, f(\tau), \lambda) \\
& =\sum_{\rho^{\prime}} f\left(\pi, \rho^{\prime}, \lambda\right)\left|\mathcal{P}_{l}^{H}\left(*, \rho^{\prime}\right)\right| .
\end{aligned}
$$

The proof of the second identity is similar.

The identities of the last proposition show that the function $f$ contains the information of the four graphs.

Corollary 5.2. For any $\gamma_{i j} \in B_{i j}$, we have:

$$
\begin{aligned}
& A_{H_{j}}\left(\gamma_{j j}, \gamma_{j, 1-j}\right)=f\left(\gamma_{j j}, \alpha_{j, 1-j}, \gamma_{j, 1-j}\right)=f\left(\gamma_{j, 1-j}, \alpha_{1-j, j}, \gamma_{j j}\right) \\
& A_{V_{j}}\left(\gamma_{j j}, \gamma_{1-j, j}\right)=f\left(\alpha_{1-j, j}, \gamma_{j j}, \gamma_{1-j, j}\right)=f\left(\alpha_{j, 1-j}, \gamma_{1-j, j}, \gamma_{j j}\right) .
\end{aligned}
$$

Proof. The first identity follows immediately from specialising the first identity of Proposition 5.1 to the case $i=j, \pi=\gamma_{j j}, \lambda=\gamma_{j, 1-j}$, (and to the case $\left.i=1-j, \pi=\gamma_{j, 1-j}, \lambda=\gamma_{j j}\right)$ and $l=1$, and noting that $\alpha_{i, 1-i}$ is the unique horizontal neighbour of $*_{i}$, and that $\mathcal{P}_{1}^{H}(u, v)=A_{H}(u, v)$ for an appropriate horizontal graph $H$.

The second identity follows analogously from the second identity of Proposition 5.1 .

Proposition 5.3. (a) For each vertex $\pi \in B_{p i}$, there exists a unique vertex $\bar{\pi} \in B_{i p}$ such that $f\left(\pi, \bar{\pi}, *_{p}\right)>0$; and, in fact, $f\left(\pi, \bar{\pi}, *_{p}\right)=1$.

(b) Further, it is true that $\overline{\bar{\pi}}=\pi$, and that for all $l, k$,

$$
\begin{aligned}
& \left|\mathcal{P}_{l}^{H}\left(*_{i}, \bar{\pi}\right)\right|=\left|\mathcal{P}_{l}^{H}\left(*_{p}, \pi\right)\right| ; \\
& \left|\mathcal{P}_{k}^{V}\left(*_{p}, \bar{\pi}\right)\right|=\left|\mathcal{P}_{k}^{V}\left(*_{i}, \pi\right)\right| .
\end{aligned}
$$

Proof. We see from Lemma 3.8 that there exists at least one vertex $\bar{\pi} \in B_{\text {ip }}$ such that $f\left(\pi, \bar{\pi}, *_{p}\right)>0$. Fix positive integers $k, l$ which are (i) both even (resp., odd) if $i=p$ (resp., $i \neq p$ ), and (ii) at least as large as the maximum distance between any two vertices on any of the four graphs. Since the graphs are connected, the assumptions (i) and (ii) show that $\mathcal{P}_{k}^{V}\left(*_{i}, \pi\right) \neq \emptyset$ and $\mathcal{P}_{l}^{H}\left(*_{i}, \rho\right) \neq \emptyset$ for all $\rho \in B_{i p}$.

Since $f(\pi, \rho, \lambda) \in \mathbb{Z}_{+}$, we deduce from Proposition 5.1 that

$$
\begin{aligned}
\left|\mathcal{P}_{l}^{H}\left(*_{p}, \pi\right)\right| & =\left|\mathcal{P}_{l}^{H}\left(\pi, *_{p}\right)\right| \\
& =\sum_{\rho} f\left(\pi, \rho, *_{p}\right)\left|\mathcal{P}_{l}^{H}\left(*_{i}, \rho\right)\right| \\
& \geq\left|\mathcal{P}_{l}^{H}\left(*_{i}, \bar{\pi}\right)\right| .
\end{aligned}
$$


On the other hand, for any $\tau \in \mathcal{P}_{l}^{H}\left(*_{i}, \bar{\pi}\right), \sigma \in \mathcal{P}_{k}^{V}\left(*_{i}, \pi\right)$, note that by 'rotational symmetry' of the connection, we have the identity

$$
A\left(\sigma, \tau, *_{p}\right)_{\sigma^{\prime}}^{\tau^{\prime}}=A\left(\sigma^{\prime o}, \tau^{\prime o}, *_{i}\right)_{\sigma^{o}}^{\tau^{o}}
$$

which implies that

$$
\begin{aligned}
& \left|\mathcal{P}_{k}^{V}\left(*_{i}, \pi\right)\right|\left|\mathcal{P}_{l}^{H}\left(*_{i}, \bar{\pi}\right)\right| f\left(\pi, \bar{\pi}, *_{p}\right) \\
& =\sum_{\sigma \in \mathcal{P}_{k}^{V}\left(*_{i}, \pi\right)} \sum_{\tau \in \mathcal{P}_{l}^{H}\left(*_{i}, \bar{\pi}\right)}\left(\sum_{\sigma^{\prime} \in \mathcal{P}_{k}^{V}\left(\bar{\pi}_{, *_{p}}\right)} \sum_{\tau^{\prime} \in \mathcal{P}_{l}^{H}\left(\pi, *_{p}\right)}\left|A\left(\sigma, \tau, *_{p}\right)_{\sigma^{\prime}}^{\tau^{\prime}}\right|^{2}\right) \\
& =\sum_{\sigma^{\prime o} \in \mathcal{P}_{k}^{V}\left(*_{p}, \bar{\pi}\right)} \sum_{\tau^{\prime o} \in \mathcal{P}_{l}^{H}\left(*_{p}, \pi\right)}\left(\sum_{\sigma^{o} \in \mathcal{P}_{k}^{V}\left(\pi, *_{i}\right)} \sum_{\tau^{o} \in \mathcal{P}_{l}^{H}\left(\bar{\pi}, *_{i}\right)}\left|A\left(\sigma^{\prime}, \tau^{\prime}, *_{i}\right)_{\sigma}^{\tau}\right|^{2}\right) \\
& =\left|\mathcal{P}_{k}^{V}\left(*_{p}, \bar{\pi}\right)\right|\left|\mathcal{P}_{l}^{H}\left(*_{p}, \pi\right)\right| f\left(\bar{\pi}, \pi, *_{i}\right) ;
\end{aligned}
$$

it follows that $f\left(\bar{\pi}, \pi, *_{i}\right)>0$. Hence, we deduce, exactly as in the case of Inequality (5.1) that we also have

$$
\left|\mathcal{P}_{l}^{H}\left(*_{i}, \bar{\pi}\right)\right| \geq\left|\mathcal{P}_{l}^{H}\left(*_{p}, \pi\right)\right|
$$

and hence that, in fact,

$$
\left|\mathcal{P}_{l}^{H}\left(*_{i}, \bar{\pi}\right)\right|=\left|\mathcal{P}_{l}^{H}\left(*_{p}, \pi\right)\right| .
$$

This equation, and the manner in which Inequality (5.1) was obtained, imply that

$$
f\left(\pi, \rho, *_{p}\right)=\delta_{\rho, \bar{\pi}},
$$

and (a) is completely proved.

The uniqueness assertion of (a), coupled with Equation (5.2), Equation (5.4) and its vertical counterpart (which is proved similarly), shows that we must have $\overline{\bar{\pi}}=\pi$, and that (b)(i) and (ii) must hold at least when $k, l$ satisfy the additional hypotheses (i) and (ii) stated in the first paragraph of the proof.

On the other hand, note that both sides of (b)(i) (resp., (ii)) are zero unless $l$ (resp., $k$ ) satisfies the condition stated in (i) of the initial paragraph and is, in addition, at least as large as the length of the shortest horizontal path joining $*_{i}$ and $\bar{\pi}$ (resp., vertical path joining $*_{i}$ and $\pi$ ). Conversely, if $k$ and $l$ satisfy the two conditions of the last sentence, then the left-side (and hence the right side) of Equation (5.2) is nonzero, and we may deduce the validity of (b)(i) and (ii) (as in the special case earlier considered).

\section{Corollary 5.4.}

$$
\mu(\pi)=\mu(\bar{\pi}) \forall \pi
$$


Proof. Let $\pi \in B_{p i}$. We consider the cases $i=p$ and $i \neq p$ separately.

Case 1. $i=p$.

Let $A$ be the matrix, with rows and columns indexed by $B_{i i}$, defined by $A(\pi, \gamma)=\left|\mathcal{P}_{2}^{H}(\pi, \gamma)\right|$. This may be viewed as the adjacency matrix of a connected graph with vertex set $B_{i i}$. The equation in Proposition 5.3(b)(i), when $l=2 n$, may be re-written as:

$$
A^{n}(*, \pi)=A^{n}(*, \bar{\pi}) \forall \pi .
$$

On the other hand, it is seen that if $v$ is the vector defined by $v(\pi)=\mu(\pi)$, then $A v=\beta^{2} v$; and it now follows from the Perron-Frobenius theorem that

$$
\lim _{n \rightarrow \infty} \frac{A^{n}}{\beta^{2 n}}=P_{v},
$$

where $P_{v}$ denotes the projection onto the one-dimensional subspace spanned by the Perron-Frobenius eigenvector $v$ of $A$. It follows therefore that, if we write $w=\sum_{\pi \in B_{i i}} \mu(\pi)^{2}$, then

$$
\begin{aligned}
\frac{\mu(\pi) \mu(*)}{w} & =P_{v}(*, \pi) \\
& =\lim _{n \rightarrow \infty} \frac{1}{\beta^{2 n}} A^{n}(*, \pi) \\
& =\lim _{n \rightarrow \infty} \frac{1}{\beta^{2 n}} A^{n}(*, \bar{\pi}) \\
& =P_{v}(*, \bar{\pi}) \\
& =\frac{\mu(\bar{\pi}) \mu(*)}{w},
\end{aligned}
$$

as desired.

Case 2. $i \neq p$.

In this case, let $A_{i}$ denote the matrix with rows and columns indexed by $B_{i, 1-i}$ and defined by $A_{i}(\pi, \gamma)=\left|\mathcal{P}_{2}^{H}(\pi, \gamma)\right|$. Note that there is an obvious bijective correspondence between $\mathcal{P}_{2 n}^{H}\left(\alpha_{i, 1-i}, \gamma\right)$ and $\mathcal{P}_{2 n+1}^{H}\left(*_{i}, \gamma\right)$, so that the equation in Proposition 5.3(b)(i), when $l=2 n+1$, may be re-written as:

$$
A_{0}^{n}\left(\alpha_{01}, \gamma\right)=A_{1}^{n}\left(\alpha_{10}, \bar{\gamma}\right)
$$

Further, since the Perron-Frobenius eigenvectors of the $A_{i}$ 's are given by the $\mu$ function, and since they both have Perron-Frobenius eigenvalue given by $\beta^{2}$, we may argue exactly as in Case 1 to obtain the desired conclusion.

Proposition 5.5. For any $\pi \in B_{p i}, \rho \in B_{i r}, \gamma \in B_{r s}, \nu \in B_{p s}$, we have

$$
\sum_{\lambda \in B_{p r}} f(\pi, \rho, \lambda) f(\lambda, \gamma, \nu)=\sum_{\kappa \in B_{i s}} f(\pi, \kappa, \nu) f(\rho, \gamma, \kappa) .
$$


Proof. To start with, fix paths $\sigma_{1} \in \mathcal{P}^{V}\left(*_{r}, \rho\right), \sigma_{2} \in \mathcal{P}^{V}\left(*_{i}, \pi\right), \tau_{1} \in$ $\mathcal{P}^{H}\left(*_{i}, \rho\right), \quad \tau_{2} \in \mathcal{P}^{H}\left(*_{r}, \gamma\right)$, and suppose $\left|\sigma_{i}\right|=k_{i}$ and $\left|\tau_{i}\right|=l_{i}$ for $i=$ 1,2 . Consider the matrix $B=\left(\left(B_{\sigma_{1}^{\prime} \sigma_{2}^{\prime}}^{\tau_{1}^{\prime} \tau^{\prime}}\right)\right)$ with rows and columns indexed by $\mathcal{P}_{l_{1}+l_{2}}^{H}(\pi, \nu)$ and $\mathcal{P}_{k_{1}+k_{2}}^{V}(\gamma, \nu)$, respectively, defined by

$$
\begin{aligned}
& B_{\sigma_{1}^{\prime} \sigma_{2}^{\prime}}^{\tau_{1}^{\prime} \tau^{\prime}}=\left(\begin{array}{rrrrl} 
& & { }_{r} & \stackrel{\tau_{2}}{\rightarrow} & \gamma \\
& \sigma_{1} \downarrow & & \downarrow \sigma_{1}^{\prime} \\
*_{i} & \stackrel{\tau_{1}}{\rightarrow} & \rho & & f\left(\sigma_{1}^{\prime}\right) \\
\sigma_{2} \downarrow & & & \\
\pi & \stackrel{\tau_{1}^{\prime}}{\rightarrow} & f\left(\tau_{1}^{\prime}\right) & \stackrel{\tau_{2}^{\prime}}{\rightarrow} & \nu
\end{array}\right) \\
& =\sum_{\widetilde{\sigma}} \sum_{\widetilde{\tau}}\left(\begin{array}{rrrrl} 
& & *_{r} & \stackrel{\tau_{2}}{\rightarrow} & \gamma \\
& \sigma_{1} \downarrow & & \downarrow \sigma_{1}^{\prime} \\
*_{i} & \stackrel{\tau_{1}}{\rightarrow} & \rho & \stackrel{\widetilde{\tau}}{\rightarrow} & f\left(\sigma_{1}^{\prime}\right) \\
\sigma_{2} \downarrow & & \widetilde{\sigma} \downarrow & & \downarrow \sigma_{2}^{\prime} \\
\pi & \stackrel{\tau_{1}^{\prime}}{\rightarrow} & f\left(\tau_{1}^{\prime}\right) & \stackrel{\tau_{2}^{\prime}}{\rightarrow} & \nu
\end{array}\right) \\
& =\sum_{\widetilde{\sigma}} \sum_{\widetilde{\tau}} W_{\tau_{1} \widetilde{\sigma}}^{\sigma_{2} \tau_{1}^{\prime}} W_{\tau_{2} \sigma_{1}^{\prime}}^{\sigma_{1} \widetilde{\tau}} W_{\widetilde{\tau} \sigma_{2}^{\prime}}^{\widetilde{\sigma} \tau_{2}^{\prime}} .
\end{aligned}
$$

We shall compute the Hilbert-Schmidt norm of $B$ in two ways. First note that

$$
\begin{aligned}
B_{\sigma_{1}^{\prime} \sigma_{2}^{\prime}}^{\tau_{1}^{\prime} \tau_{2}^{\prime}} & =\sum_{\widetilde{\sigma}} \sum_{\widetilde{\tau}} W_{\tau_{1} \widetilde{\sigma}}^{\sigma_{2} \tau_{1}^{\prime}} W_{\tau_{2} \sigma_{1}^{\prime}}^{\sigma_{1} \widetilde{\tau}} W_{\widetilde{\tau} \sigma_{2}^{\prime}}^{\widetilde{\sigma} \tau_{2}^{\prime}} \\
& =\sum_{\widetilde{\sigma}} A\left(\sigma_{2}, \tau_{1}, f\left(\tau_{1}^{\prime}\right)\right)_{\widetilde{\sigma}}^{\tau_{1}^{\prime}} A\left(\sigma_{1} \widetilde{\sigma}, \tau_{2}, \nu\right)_{\sigma_{1}^{\prime} \sigma_{2}^{\prime}}^{\tau_{2}^{\prime}}
\end{aligned}
$$

and that also

$$
\begin{aligned}
B_{\sigma_{1}^{\prime} \sigma_{2}^{\prime}}^{\tau_{1}^{\prime} \tau_{2}^{\prime}} & =\sum_{\widetilde{\sigma}} \sum_{\widetilde{\tau}} W_{\tau_{1} \widetilde{\sigma}}^{\sigma_{2} \tau_{1}^{\prime}} W_{\tau_{2} \sigma_{1}^{\prime}}^{\sigma_{1} \widetilde{\tau}} W_{\widetilde{\tau} \sigma_{2}^{\prime}}^{\widetilde{\sigma} \tau^{\prime}} \\
& =\sum_{\widetilde{\tau}} A\left(\sigma_{1}, \tau_{2}, f\left(\sigma_{1}^{\prime}\right)\right)_{\sigma_{1}^{\prime}}^{\widetilde{\tau}} A\left(\sigma_{2}, \tau, \widetilde{\tau}, \nu\right)_{\sigma_{2}^{\prime}}^{\tau_{1}^{\prime} \tau_{2}^{\prime}}
\end{aligned}
$$

Deduce from Equation (5.7) that

$$
\begin{aligned}
& \left|\begin{array}{l}
B_{\sigma_{1}^{\prime} \sigma_{2}^{\prime}}^{\tau_{1}^{\prime} \tau_{2}^{\prime}} \\
\mid
\end{array}\right|=\left|\sum_{\widetilde{\sigma}} A\left(\sigma_{2}, \tau_{1}, f\left(\tau_{1}^{\prime}\right)\right)_{\widetilde{\sigma}}^{\tau_{1}^{\prime}} A\left(\sigma_{1} \widetilde{\sigma}, \tau_{2}, \nu\right)_{\sigma_{1}^{\prime} \sigma_{2}^{\prime}}^{\tau^{\prime}}\right|^{2} \\
& =\sum_{\widetilde{\sigma}, \widetilde{\sigma}_{1}} A\left(\sigma_{2}, \tau_{1}, f\left(\tau_{1}^{\prime}\right)\right)_{\widetilde{\sigma}}^{\tau_{1}^{\prime}} A\left(\sigma_{1} \widetilde{\sigma}, \tau_{2}, \nu\right)_{\sigma_{1}^{\prime} \sigma_{2}^{\prime}}^{\tau_{2}^{\prime}} \frac{A\left(\sigma_{2}, \tau_{1}, f\left(\tau_{1}^{\prime}\right)\right)_{\widetilde{\sigma}_{1}}^{\tau_{1}^{\prime}} A\left(\sigma_{1} \widetilde{\sigma}_{1}, \tau_{2}, \nu\right)_{\sigma_{1}^{\prime} \sigma_{2}^{\prime}}^{\tau^{\prime}}}{}
\end{aligned}
$$


and hence,

$$
\begin{aligned}
& \|B\|_{2}^{2}=\sum_{\tau_{1}^{\prime} \tau_{2}^{\prime}} \sum_{\sigma_{1}^{\prime} \sigma_{2}^{\prime}}\left|B_{\sigma_{1}^{\prime} \sigma_{2}^{\prime}}^{\tau_{1}^{\prime} \tau^{\prime}}\right|^{2} \\
& =\sum_{\tau_{1}^{\prime} \tau_{2}^{\prime}} \sum_{\sigma_{1}^{\prime} \sigma_{2}^{\prime}} \sum_{\widetilde{\sigma}, \widetilde{\sigma}_{1}} A\left(\sigma_{2}, \tau_{1}, f\left(\tau_{1}^{\prime}\right)\right)_{\widetilde{\sigma}}^{\tau_{1}^{\prime}} \overline{A\left(\sigma_{2}, \tau_{1}, f\left(\tau_{1}^{\prime}\right)\right)_{\widetilde{\sigma}_{1}}^{\tau_{1}^{\prime}}} \\
& \cdot A\left(\sigma_{1} \tilde{\sigma}, \tau_{2}, \nu\right)_{\sigma_{1}^{\prime} \sigma_{2}^{\prime}}^{\tau_{2}^{\prime}} \overline{A\left(\sigma_{1} \widetilde{\sigma}_{1}, \tau_{2}, \nu\right)_{\sigma_{1}^{\prime} \sigma_{2}^{\prime}}^{\tau^{\prime}}} \\
& =\sum_{\tau_{1}^{\prime} \tau_{2}^{\prime} \widetilde{\sigma}_{,}, \sigma_{1}} A\left(\sigma_{2}, \tau_{1}, f\left(\tau_{1}^{\prime}\right)\right)_{\widetilde{\sigma}}^{\tau_{1}^{\prime}} \overline{A\left(\sigma_{2}, \tau_{1}, f\left(\tau_{1}^{\prime}\right)\right)_{\widetilde{\sigma}_{1}}^{\tau_{1}^{\prime}}} \\
& \cdot \sum_{\sigma_{1}^{\prime} \sigma_{2}^{\prime}} A\left(\sigma_{1} \widetilde{\sigma}, \tau_{2}, \nu\right)_{\sigma_{1}^{\prime} \sigma_{2}^{\prime}}^{\tau_{2}^{\prime}} \overline{A\left(\sigma_{1} \widetilde{\sigma}_{1}, \tau_{2}, \nu\right)_{\sigma_{1}^{\prime} \sigma_{2}^{\prime}}^{\tau^{\prime}}} \\
& =\sum_{\tau_{1}^{\prime} \tau_{2}^{\prime}} \sum_{\widetilde{\sigma}, \widetilde{\sigma}_{1}} A\left(\sigma_{2}, \tau_{1}, f\left(\tau_{1}^{\prime}\right)\right)_{\widetilde{\sigma}}^{\tau_{1}^{\prime}} \overline{A\left(\sigma_{2}, \tau_{1}, f\left(\tau_{1}^{\prime}\right)\right)_{\widetilde{\sigma}_{1}^{\prime}}^{\tau_{1}^{\prime}}} \\
& \cdot\left(A\left(\sigma_{1} \widetilde{\sigma}, \tau_{2}, \nu\right) A\left(\sigma_{1} \widetilde{\sigma}_{1}, \tau_{2}, \nu\right)^{*}\right)_{\tau_{2}^{\prime}}^{\tau_{2}^{\prime}} \\
& =\sum_{\tau_{1}^{\prime}} \sum_{\widetilde{\sigma}, \widetilde{\sigma}_{1}} A\left(\sigma_{2}, \tau_{1}, f\left(\tau_{1}^{\prime}\right)\right)_{\widetilde{\sigma}}^{\tau_{1}^{\prime}} \overline{A\left(\sigma_{2}, \tau_{1}, f\left(\tau_{1}^{\prime}\right)\right)_{\widetilde{\sigma}_{1}}^{\tau_{1}^{\prime}}} \\
& \cdot \operatorname{Tr}\left(\delta_{\sigma_{1} \widetilde{\sigma}_{,} \sigma_{1} \widetilde{\sigma}_{1}} V\left(\tau_{2}, \tau_{2}, f\left(\tau_{1}^{\prime}\right), \nu\right)\right) \\
& =\sum_{\tau_{1}^{\prime}} \sum_{\widetilde{\sigma}}\left|A\left(\sigma_{2}, \tau_{1}, f\left(\tau_{1}^{\prime}\right)\right)_{\widetilde{\sigma}}^{\tau_{1}^{\prime}}\right|^{2} f\left(f\left(\tau_{1}^{\prime}\right), \gamma, \nu\right) \\
& =\sum_{\lambda \in B_{p r}} f(\pi, \rho, \lambda) f(\lambda, \gamma, \nu) .
\end{aligned}
$$

An entirely similar argument, starting with Equation (5.8) rather than (5.7), shows that

$$
\|B\|_{2}^{2}=\sum_{\kappa \in B_{i s}} f(\pi, \kappa, \nu) f(\rho, \gamma, \kappa),
$$

and the proof of the proposition is complete.

Proposition 5.6. For any $\pi \in B_{p i}, \rho \in B_{i r}, \lambda \in B_{p r}$, we have:

$$
f(\pi, \rho, \lambda)=f(\bar{\pi}, \lambda, \rho) .
$$

Proof. Much of the following notation will become clearer if one keeps the following diagram in mind:

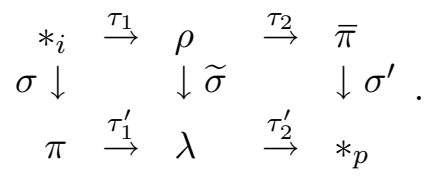


Choose positive integers $k, l_{1}, l_{2}$ such that (a) $k-(p-i), l_{1}-(r-i)$ and $l_{2}-(p-r)$ are all even, and (b) each of $k, l_{1}$ and $l_{2}$ are at least as large as the maximum of the diameters of the four graphs. It then follows that all the following sets are nonempty: $\mathcal{P}_{k}^{V}\left(*_{i}, \pi\right), \mathcal{P}_{k}^{V}(\rho, \lambda), \mathcal{P}_{k}^{V}\left(*_{p}, \bar{\pi}\right), \mathcal{P}_{l_{1}}^{H}\left(*_{i}, \rho\right), \mathcal{P}_{l_{1}}^{H}(\pi, \lambda), \mathcal{P}_{l_{2}}^{H}(\rho, \bar{\pi}), \mathcal{P}_{l_{2}}^{H}\left(\lambda, *_{p}\right)$.

Let us write $l=l_{1}+l_{2}$. We will need the admissible tuples $(\mathbf{k}, \mathbf{l})=$ $((k),(l)), \quad\left(\mathbf{k}^{\prime}, \mathbf{l}^{\prime}\right)=\left((0, k),\left(l_{1}, l_{2}\right)\right),\left(\mathbf{k}^{\prime \prime}, \mathbf{l}^{\prime \prime}\right)=((0, k),(l, 0))$, and the associated unitary matrices $W=W_{(\mathbf{k}, \mathbf{l}),\left(\mathbf{k}^{\prime \prime}, \mathbf{l}^{\prime \prime}\right)}\left(*_{i}, *_{p}\right), W_{1}=W_{(\mathbf{k}, \mathbf{l}),\left(\mathbf{k}^{\prime}, \mathbf{l}^{\prime}\right)}\left(*_{i}, *_{p}\right)$, and $W_{2}=W_{\left(\mathbf{k}^{\prime}, \mathbf{l}^{\prime}\right),\left(\mathbf{k}^{\prime \prime}, \mathbf{l}^{\prime \prime}\right)}\left(*_{i}, *_{p}\right)$. Then, it follows from Lemma 2.3 that $W=$ $W_{1} W_{2}$.

Let us write $P_{\pi}$ (resp., $R_{\bar{\pi}}$ ) for the diagonal matrix with rows and columns indexed by paths of type $(\mathbf{k}, \mathbf{l})$ (resp., $\left.\left(\mathbf{k}^{\prime \prime}, \mathbf{l}^{\prime \prime}\right)\right)$ from $*_{i}$ to $*_{p}$, which represents the projection onto the subspace spanned by $\mathcal{P}_{\pi}=\left\{\sigma \tau^{\prime} \in \mathcal{P}_{k l}^{V H}\left(*_{i}, *_{p}\right), f(\sigma)\right.$ $=\pi\}$ (resp., $\mathcal{P}_{\bar{\pi}}=\left\{\tau \sigma^{\prime} \in \mathcal{P}_{l k}^{H V}\left(*_{i}, *_{p}\right), f(\tau)=\bar{\pi}\right\}$ ). Then, thanks to Proposition 5.3, we see that $U=P_{\pi} W R_{\bar{\pi}}$ is unitary when viewed as a matrix with rows and columns indexed by $\mathcal{P}_{\pi}$ and $\mathcal{P}_{\bar{\pi}}$ respectively. Then, $U=A B$, where $A=P_{\pi} W_{1}$ (resp. $B=W_{2} R_{\bar{\pi}}$ ) is the rectangular matrix obtained by suitably restricting the rows of $W_{1}$ (resp., columns of $W_{2}$ ).

Since $A$ is obtained by taking some collection of rows of a unitary matrix, it is seen that $A$ is a coisometry. Similarly, it is seen that $B$ is an isometry. Hence, $U=A B$ is a factorisation of a unitary matrix as a product of a coisometry and an isometry. We can deduce from this that we must have $A^{*} A=B B^{*}$ and that hence

$$
U B^{*}=A B B^{*}=A A^{*} A=A .
$$

Now let $Q_{\rho \lambda}$ be the diagonal matrix with rows and columns indexed by paths of type $\left(\mathbf{k}^{\prime}, \mathbf{l}^{\prime}\right)$ from $*_{i}$ to $*_{p}$, which represents the projection onto the subspace spanned by the set $\mathcal{P}_{\rho \lambda}$ consisting of all paths $\tau_{1} \widetilde{\sigma} \tau_{2}^{\prime}$ of type $\left(\mathbf{k}^{\prime}, \mathbf{l}^{\prime}\right)$ which satisfy $f\left(\tau_{1}\right)=\rho$ and $f(\widetilde{\sigma})=\lambda$.

Then, we have

$$
A Q_{\rho \lambda}=U B^{*} Q_{\rho \lambda}=U\left(Q_{\rho \lambda} B\right)^{*}
$$

and hence

$$
\left\|A Q_{\rho \lambda}\right\|_{2}=\left\|U\left(Q_{\rho \lambda} B\right)^{*}\right\|_{2}=\left\|\left(Q_{\rho \lambda} B\right)^{*}\right\|_{2}=\left\|Q_{\rho \lambda} B\right\|_{2} .
$$

Now, on the one hand, we have

$$
\begin{aligned}
\left\|A Q_{\rho \lambda}\right\|_{2}^{2} & =\sum_{\sigma, \tau_{1}^{\prime}, \tau_{1}, \widetilde{\sigma}, \tau_{2}^{\prime}}\left\|\begin{array}{ccccc}
*_{i} & \stackrel{\tau_{1}}{\rightarrow} & \rho & \\
\sigma \downarrow & & \downarrow & \\
\pi & \stackrel{\tau_{1}^{\prime}}{\rightarrow} & \lambda & \stackrel{\tau_{2}^{\prime}}{\rightarrow} & *_{p}
\end{array}\right\|_{2}^{2} \\
& =\left|\mathcal{P}_{l_{2}}^{H}\left(\lambda, *_{p}\right)\right|\left|\mathcal{P}_{k}^{V}\left(*_{i}, \pi\right)\right|\left|\mathcal{P}_{l_{1}}^{H}\left(*_{i}, \rho\right)\right| f(\pi, \rho, \lambda) ;
\end{aligned}
$$


while on the other hand,

$$
\begin{aligned}
& \left\|Q_{\rho \lambda} B\right\|_{2}^{2}=\sum_{\tau_{1}, \tilde{\sigma}, \tau_{2}^{\prime}, \tau_{2}, \sigma^{\prime}}\left\|\begin{array}{ccccc}
*_{i} & \stackrel{\tau_{1}}{\rightarrow} & \rho & \stackrel{\tau_{2}}{\rightarrow} & \bar{\pi} \\
& \tilde{\sigma} \downarrow & & \downarrow \sigma^{\prime} \\
& \lambda & \stackrel{\tau_{2}^{\prime}}{\rightarrow} & *_{p}
\end{array}\right\|_{2}^{2} \\
& =\left|\mathcal{P}_{l_{1}}^{H}\left(*_{i}, \rho\right)\right|\left|\mathcal{P}_{k}^{V}\left(\bar{\pi}, *_{p}\right)\right|\left|\mathcal{P}_{l_{2}}^{H}\left(\lambda, *_{p}\right)\right| f(\bar{\pi}, \lambda, \rho) ;
\end{aligned}
$$

and the desired conclusion follows from Equations (5.11), (5.12) and (5.13) (and from Equation (5.4)).

We may now deduce, from Propositions 5.5 and 5.6, that each $B_{i i}$ may be regarded as the basis of a fusion algebra with the structure constants (i.e., fusion coefficients) being given by $\langle\pi \otimes \rho, \lambda\rangle=f(\pi, \rho, \lambda)$ and the involution being given by $\pi \mapsto \bar{\pi}$. From general facts - see [VS], for instance - about such fusion algebras, we see that we also have, in addition to Equation (5.9), the following identities:

$$
f(\pi, \rho, \lambda)=f(\lambda, \bar{\rho}, \pi)=f(\bar{\rho}, \bar{\pi}, \bar{\lambda}) .
$$

(Strictly speaking, the general facts described in [VS] will only yield Equation (5.14) in the special case where all the three vertices belong to the same $B_{i i}$; but a minor extension of the arguments there show that these equations are valid in total generality, i.e., whenever $\pi \in B_{p i}, \rho \in B_{i r}, \lambda \in B_{p r}$.)

In particular, if $\pi \in B_{j j}$ and $\lambda \in B_{j, 1-j}$, and if we set $\rho=\alpha_{j, 1-j}$, we may deduce from Equation (5.14) and Corollary 5.2 that

$$
\begin{aligned}
A_{H_{j}}(\pi, \lambda) & =f\left(\pi, \alpha_{j, 1-j}, \lambda\right) \\
& =f\left(\bar{\alpha}_{j, 1-j}, \bar{\pi}, \bar{\lambda}\right) \\
& =f\left(\alpha_{1-j, j}, \bar{\pi}, \bar{\lambda}\right) \\
& =A_{V_{j}}(\bar{\pi}, \bar{\lambda}) .
\end{aligned}
$$

The truth of the following corollary is immediate.

Corollary 5.7. The mapping $\gamma \mapsto \bar{\gamma}$ establishes an isomorphism between the graphs $V_{j}$ and $H_{j}$, for $j=1,2$.

\section{References}

[DB] D. Bisch, On the structure of finite depth subfactors, Algebraic Methods in Operator Theory, Birkhaüser, Boston, 1994, 175-194, MR 95j:46073, Zbl 809.46069.

[DB1] _ Bimodules, higher relative commutants and the fusion algebra associated to a subfactor, Operator algebras and their applications, Fields Institute Communications, 13, American Math. Soc., (1997), 13-63, MR 97i:46109, Zbl 894.46046.

[EK] D.E. Evans and Y. Kawahigashi, Quantum Symmetries on Operator Algebras, Oxford University Press, 1998, MR 99m:46148, Zbl 924.46054. 
[FRS] K. Fredenhagen, K.H. Rehren and B. Schroer, Superselection sectors with braid group statistics and exchange algebras I. General theory, Comm. in Math. Physics, 125 (1989), 201-226, MR 91c:81047, Zbl 682.46051.

[FRG] F.R. Gantmacher, Theory of Matrices, Vol. 2, Chelsea, 1959, MR 21 \#6372c, Zbl 085.01001.

[Gne] Sante Gnerre, An explicit formula for fusion rules, Internat. J. Math., 1 (2002), $1-8$.

[GW] F. Goodman and H. Wenzl, Littlewood Richardson coefficients for Hecke algebras at roots of unity, Adv. in Math., 82 (1990), 244-265, MR 91i:20013, Zbl 714.20004.

[Iz] M. Izumi, Application of fusion rules to classification of subfactors, Publications of the RIMS, Kyoto University, 27 (1991), 953-994, MR 93b:46121, Zbl 765.46048.

[VJ] V. Jones, Index for subfactors, Invent. Math., 72 (1983), 1-25, MR 84d:46097, Zbl 508.46040.

[JS] V. Jones and V.S. Sunder, Introduction to Subfactors, London Math. Soc. Lecture Note Series, 234, Cambridge University Press, Cambridge, 1997, MR 98h:46067, Zbl 903.46062.

[YK] Y. Kawahigashi, On flatness of Ocneanu's connections on the Dynkin diagrams and classification of subfactors, J. of Funct. Analy., 127 (1995), 63-107, MR 95j:46075, Zbl 829.46048.

[Lo] R. Longo, Index of subfactors and statistics of quantum fields, I, Comm. in Math. Physics, 126, (1989) 217-247, MR 91c:46097, Zbl 682.46045.

[Lo1] Index of subfactors and statistics of quantum fields, II, Comm. in Math. Physics, 130 (1990), 285-309, MR 91j:46083, Zbl 705.46038.

[AO1] A. Ocneanu, Quantized groups, string algebras and Galois theory for algebras, Operator Algebras and Appl., Vol. 2 (Warwick 1987), London Math. Soc. Lecture Notes Ser., Vol. 136, Cambridge University Press, Cambridge, 1988, 119-172, MR 91k:46068, Zbl 696.46048.

[AO2] A. Ocneanu, (Lecture Notes written by Y. Kawahigashi), Quantum Symmetry, Differential Geometry of Finite Graphs, and Classification of Subfactors, Univ. of Tokyo Seminar Notes, Tokyo, 1990.

[VS] V.S. Sunder, $\mathrm{II}_{1}$ factors, their bimodules and hypergroups, Trans. of the Amer. Math. Soc., 330 (1992), 227-256, MR 92f:46076, Zbl 757.46053.

[SV] V.S. Sunder and A.K. Vijayarajan, On the non-occurrence of the Coxeter graphs $\beta_{2 n+1}, D_{2 n+1}$ and $E_{7}$ as the principal graph invariant of an inclusion of $\mathrm{II}_{1}$ factors, Pacific J. Math., 161(1) (1993), 185-200, MR 94g:46067, Zbl 798.43005.

Received November 10, 1999 and revised July 31, 2000.

The Institute of Mathematical Sciences

Chennai 600113

INDIA

E-mail address: vijay@imsc.ernet.in

The Institute of Mathematical Sciences

Chennai 600113

INDIA

E-mail address: sunder@imsc.ernet.in 